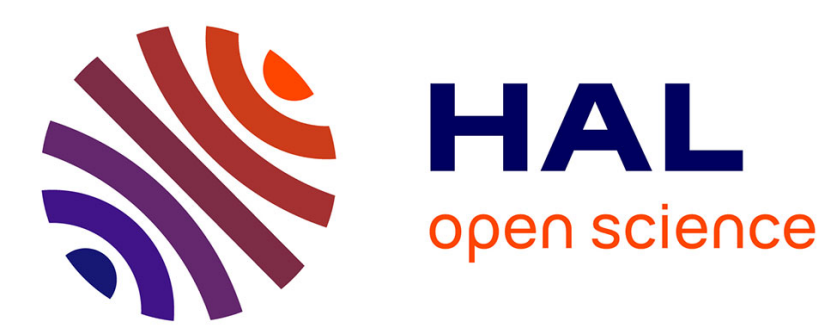

\title{
A reduced fracture model for two-phase flow with different rock types
}

\author{
Elyes Ahmed, Jérôme Jaffré, Jean E. Roberts
}

\section{To cite this version:}

Elyes Ahmed, Jérôme Jaffré, Jean E. Roberts. A reduced fracture model for two-phase flow with different rock types. Mathematics and Computers in Simulation, 2015. hal-01119986v2

\section{HAL Id: hal-01119986 \\ https://hal.inria.fr/hal-01119986v2}

Submitted on 6 Feb 2017

HAL is a multi-disciplinary open access archive for the deposit and dissemination of scientific research documents, whether they are published or not. The documents may come from teaching and research institutions in France or abroad, or from public or private research centers.
L'archive ouverte pluridisciplinaire HAL, est destinée au dépôt et à la diffusion de documents scientifiques de niveau recherche, publiés ou non, émanant des établissements d'enseignement et de recherche français ou étrangers, des laboratoires publics ou privés. 


\title{
A reduced fracture model for two-phase flow with different rock types
}

\author{
Elyes Ahmed ${ }^{\mathrm{a}, *}$, Jérôme Jaffréb ${ }^{\mathrm{b}}$, Jean E. Roberts ${ }^{\mathrm{b}}$ \\ ${ }^{a}$ Université de Tunis El Manar, ENIT-LAMSIN, BP 37, 1002, Tunis le Belvédère,Tunisia \\ ${ }^{b}$ INRIA Paris-Rocquencourt, 78153 Le Chesnay cedex, France
}

\begin{abstract}
This article is concerned with the numerical discretization of a model for incompressible twophase flow in a porous medium with fractures. The model is a discrete fracture model in which the fractures are treated as interfaces of dimension 2 in a 3-dimensional simulation, with fluid exchange between the 2-dimensional fracture flow and the 3-dimensional flow in the surrounding rock matrix. The model takes into account the change in the relative permeabilities and in the capillary pressure curves which occurs at the interface between the fracture and the rock matrix. The model allows for barriers which are fractures with low permeability. Mixed finite elements and advective upstream weighting are used to discretize the problem and numerical experiments are shown.

Keywords: Flow in porous media, Discrete fracture model, Two-phase flow, Discontinuous capillary pressure, Mixed finite elements,
\end{abstract}

\section{Introduction}

The presence of fractures in a porous medium greatly complicates the modeling of flow and transport in a porous medium. Fractures occur on different scales, with different geometries, and may behave either as channels or as barriers for the fluid flow. The fractures thus have a very strong influence on flow and transport, either making flow in certain directions several orders of magnitude more rapid than in other directions or possibly nearly blocking flow in certain directions. There is a need for complex simulation models that resolve the flow dynamics

\footnotetext{
This research was partially funded by the Hydrinv EuroMed project

*Principal corresponding author. Present address: INRIA Paris-Rocquencourt, 78153 Le Chesnay cedex, France.

Email addresses: elyes . ahmed@inria.fr (Elyes Ahmed), jerome.jaffre@inria.fr (Jérôme Jaffré), jean.roberts@inria.fr (Jean E. Roberts)

Preprint submitted to Mathematics and Computers in Simulation 
along fractures and the fluid interaction with the porous matrix. The difficulties in the numerical modeling of multiphase flow in fractured media stem from the extremely heterogeneous and anisotropic fracture matrix system and from the nonlinearity due the relative permeability and capillary pressure. Thus some special method for dealing with these difficulties is required. The so called continuum models take fractures into account through a sort of averaging or homogenizing process. Discrete fracture models include the fractures individually in the model. Often in these models it is considered that the amount of flow outside the fractures is negligible and flow in the domain is modeled as flow in a network of fractures which don't communicate with the surrounding medium. More complex discrete fracture models take into account exchange between fractures and the surrounding medium. This last type of model is that of interest for us here, and more precisely we are concerned with reduced fracture models that treat fractures as $(n-1)$-dimensional objects embedded in an $n$-dimensional medium, $n$ being either 2 or 3 , and in which there is communication between the $n$-dimensional and $(n-1)$ - dimensional mediums. A number of articles have been written on numerical models of this type for one-phase flow; see for example $[10,3,11,4,37,8,26,40,23,45,21,20]$ and references therein. For two-phase flow the situation is more complicated due to the change of capillary pressure and relative permeability curves between the fracture and the matrix rock [35, 12, 33, 43, 39, 30, 24, 16, 42]. In [32] a reduced fracture model which uses the global pressure formulation was introduced which can treat the case of barriers as well as the case of fractures with larger permeability than in the matrix rock, as was done for Darcy flow in [37]. In this paper we present a discretization method of that model using mixed finite elements and show numerical experiments.

After this introduction we recall briefly in Section 2 the global pressure formulation for incompressible two-phase flow. In Section 3 we present a model for incompressible two-phase flow in a fractured domain for which the fracture is modeled as a thin layer in the surrounding matrix, and in Section 4 we show how the reduced model, which treats the fracture as an interface, is obtained. The case of intersecting fractures is considered in Section 5. A multidomain formulation for the reduced model is introduced in Section 6. Numerical methods are discussed in Section 7 and some comments on implementation are made in Section 8. Section 9 shows some numerical experiments. 


\section{The global pressure formulation for incompressible two-phase flow}

We consider incompressible two-phase flow in a porous medium. The governing equations are the equations expressing volume conservation (or equivalently mass conservation since the fluids are assumed to be incompressible) of the two fluid phases and the Darcy law for each phase. For the $\ell$ phase, $\ell=w$ (wetting phase) or $n w$ (nonwetting phase) the phase conservation equation is

$$
\Phi \frac{\partial s_{\ell}}{\partial t}+\nabla \cdot \mathbf{u}_{\ell}=q_{\ell}, \quad \ell \in\{w, n w\}
$$

where $s_{\ell}$ is the saturation of the $\ell$ phase, $\mathbf{u}_{\ell}$ is its volumetric flow rate, i.e. its Darcy velocity, $q_{\ell}$ is the source term given as a function of the phase saturation $\left(0 \leq s_{\ell} \leq 1\right)$ and $\Phi$ is the porosity of the domain. We assume that the volume of all pores is filled by the two phases so that

$$
s_{n}+s_{n w}=1
$$

and we choose for the main saturation unknown the saturation of the wetting phase $s=s_{w}$.

The Darcy velocity of the $\ell$ phase $\mathbf{u}_{\ell}$ is related to the phase pressure $p_{\ell}$ by Darcy's law:

$$
\mathbf{u}_{\ell}=-\mathbf{K} k_{\ell}(s)\left(\nabla p_{\ell}-\rho_{\ell} \mathbf{u}_{G}\right), \quad \ell \in\{w, n w\},
$$

where $\mathbf{K}$ denotes the tensor field of absolute permeability, a bounded symmetric uniformly positive definite matrix. $\rho_{\ell}$ and $k_{\ell}$ are respectively the phase density and the phase mobility, and $\mathbf{u}_{G}$ denotes the gravity field. The mobilities are positive monotone functions of the saturation $s: k_{w}$ is increasing with $s=s_{w}$ and $k_{w}(0)=0$, while $k_{n w}$ is decreasing with $s=1-s_{n w}$ and $k_{n w}(1)=0$. Equations (1) and (3) taken together constitute the $\ell$-phase saturation equation written in mixed form.

The difference between the phase pressures is the capillary pressure $\pi$ :

$$
\pi(s)=p_{n w}-p_{w},
$$

which is a positive, decreasing function of $s$. The capillary pressure curve $\pi$ and the relative permeability curves $k_{\ell}, \ell=w, n w$, depend on the physical properties of the two phases and those of the rock. Among the most frequently used models for describing capillary pressure and relative permeabilities as functions of the saturation are those given by Van Genuchten [17] and that given by Brooks and Corey $[31,39]$. Further detail concerning capillary pressure and relative permeability can be found in $[46,18]$. 
To introduce the global pressure formulation we follow the development in [18]. We use the total velocity $\mathbf{u}$ which is the sum of the two Darcy velocities, that of the wetting phase and that of the nonwetting phase:

$$
\mathbf{u}=\mathbf{u}_{w}+\mathbf{u}_{n w}=-\sum_{\ell \in\{w, n w\}} \mathbf{K} k_{\ell}(s)\left(\nabla p_{\ell}-\rho_{\ell} \mathbf{u}_{G}\right),
$$

and adding the two phase conservation equations (1), for $\ell \in\{w, n w\}$, and using (2), we may write

$$
\nabla \cdot \mathbf{u}=q_{n w}(1-s)+q_{w}(s)=q(s) .
$$

The idea for the global pressure is to introduce a quantity, the "global pressure", so that we can write a "Darcy-type" equation which gives the total velocity in terms of this "global pressure". The global pressure $p$ should meet the following criteria:

- $p_{w} \leq p \leq p_{n w}$

- if $s=1$ then $p=p_{w}$;

Toward the end of defining $p$ we introduce the fractional flow curve for each phase, $\ell=w, n w$,

$$
f_{\ell}(s)=\frac{k_{\ell}(s)}{k(s)}, \quad \text { where } k(s)=k_{w}(s)+k_{n w}(s),
$$

and define a weighted average density $\rho$, weighted by the fractional flow:

$$
\rho(s)=f_{w}(s) \rho_{w}+f_{n w}(s) \rho_{n w} .
$$

Now the global pressure is defined by

$$
p=\frac{1}{2}\left(p_{w}+p_{n w}\right)+\beta(s),
$$

where $\beta$ is the function $\beta(s)=\int_{1}^{s}\left(\frac{1}{2}-f_{w}(\sigma)\right) \pi^{\prime}(\sigma) d \sigma$. It was shown in [18] that the total velocity and the global pressure, as defined in (9), are related by the following Darcy-type law:

$$
\mathbf{u}=-\mathbf{K} k(s)\left(\nabla p-\rho(s) \mathbf{u}_{G}\right) .
$$

We stress the fact that the global pressure is not a physical pressure and is only a mathematical tool. It is a smooth function defined in the whole domain, whether a phase vanishes or not. Note also that using equations (4) and (9) we can write the phase pressures in terms of the capillary pressure and the global pressure:

$$
p_{w}=p-\beta(s)-\frac{1}{2} \pi(s), \quad p_{4}=p-\beta(s)+\frac{1}{2} \pi(s) .
$$


Then the pressure equation (in mixed form) is given as follows:

$$
\begin{aligned}
& \nabla \cdot \mathbf{u}=q(s), \\
& \mathbf{u}=-\mathbf{K} k(s)\left(\nabla p-\rho(s) \mathbf{u}_{G}\right) .
\end{aligned}
$$

This pressure equation together with the saturation equation for the wetting phase fluid will form the system for the global pressure formulation. However, still following [18], we will rewrite each of the Darcy phase velocities (3) as the sum of a diffusion term and an advection term. To do so we first introduce, for $\ell \in\{w, n w\}$, the nonlinear functions

$$
f_{G \ell}(s)=k_{\ell}(s)\left(\rho_{\ell^{\prime}}-\rho_{\ell}\right), \text { where } \ell^{\prime} \in\{w, n w\}, \ell^{\prime} \neq \ell .
$$

and then introduce the Kirchoff transform

$$
a(s)=-\frac{k_{w} k_{n w}}{k_{w}+k_{n w}} \pi^{\prime}(s), \quad \alpha(s)=\int_{0}^{s} a(\sigma) d \sigma .
$$

Note that $a(0)=a(1)=0$ and that $\alpha(0)=0$.

Now the Darcy velocity of the $\ell$-phase can be written as follows

$$
\mathbf{u}_{\ell}=\iota_{\ell} \mathbf{K} \nabla \alpha(s)+f_{\ell}(s)\left(\mathbf{u}+f_{G \ell}(s) \mathbf{K u}_{G}\right),
$$

where $\iota_{\ell}=-1$ if $\ell=w$ and $\iota_{\ell}=1$ if $\ell=n w$. Thus, for the wetting phase, $\mathbf{u}_{w}$ is the sum of a capillary diffusion term $\mathbf{r}=-\mathbf{K} \nabla \alpha(s)$, and of an advection term $\mathbf{f}=f_{w}(s)\left(\mathbf{u}+f_{G w}(s) \mathbf{K u}_{G}\right)$.

The (wetting-phase) saturation equation can now be written as

$$
\begin{aligned}
& \Phi \frac{\partial s}{\partial t}+\nabla \cdot \mathbf{u}_{w}=q_{w}, \quad \mathbf{u}_{w}=\mathbf{r}+\mathbf{f}, \\
& \mathbf{r}=-\mathbf{K} \nabla \alpha(s), \quad \mathbf{f}=f_{w}(s)\left(\mathbf{u}+f_{G w}(s) \mathbf{K} \mathbf{u}_{G}\right) .
\end{aligned}
$$

\section{Two-phase flow in a domain with a fracture}

We identify the porous medium with a domain $\Omega \subset \mathbb{R}^{3}$ and suppose that it can be divided into 3 non overlapping, connected subdomains, $\Omega_{1}, \Omega_{f}$ and $\Omega_{2}$, with $\bar{\Omega}=\bar{\Omega}_{1} \cup \bar{\Omega}_{f} \cup \bar{\Omega}_{2}$ and $\bar{\Omega}_{1} \cap \bar{\Omega}_{2}=\emptyset$; see Fig 1 . The subdomains $\Omega_{1}$ and $\Omega_{2}$ represent the matrix rock while $\Omega_{f}$ represents a fracture supposed also to be a porous medium. The fracture $\Omega_{f}$ is delimited by two surfaces $\gamma_{i}=\partial \Omega_{f} \cap \partial \Omega_{i}, i=1,2$. Its width $d$ is very small compared to the size of the whole domain $\Omega$. For simplicity we suppose in fact that $\Omega_{f}$ is of the form $\gamma \times\left(-\frac{d}{2}, \frac{d}{2}\right)$ where $\gamma$ is the intersection 


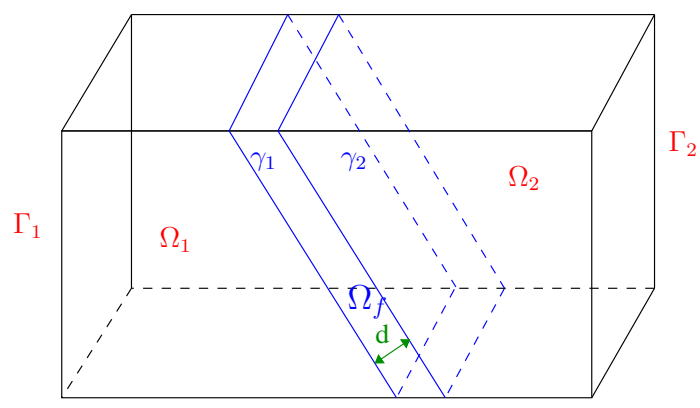

Figure 1: The domain $\Omega$ with the physical fracture

of a hyperplane with $\Omega$ so that each element of $\Omega_{f}$ may be written uniquely in the form $\mathbf{x}+\xi \mathbf{n}$ where $\mathbf{x} \in \gamma, \xi \in\left(-\frac{d}{2}, \frac{d}{2}\right)$ and $\mathbf{n}$ is a unit vector normal to $\gamma$.

The matrix rock and the fracture are assumed to be of different rock types, which implies not only that the porosity and the absolute permeability may differ but also that the nonlinear functions of the saturation, such as the capillary pressure curve and the relative permeability curves may not be the same in the fracture and in the rock matrix. Let $\Gamma=\partial \Omega$ and let $\Gamma^{p D}$ and $\Gamma^{p N}$ denote the Dirichlet and Neumann, boundaries, respectively, of $\Omega$ for the pressure equation while $\Gamma^{s D}$ and $\Gamma^{s N}$ denote the Dirichlet and Neumann boundaries, respectively, of $\Omega$ for the saturation equation. For each of these we will use an index $i$ to denote its intersection with $\partial \Omega_{i}: i=1,2$ or $f$. We will denote by $\Gamma_{i}^{e B}$ the intersection $\Gamma_{i}^{e B}=\Gamma^{e B} \cap \partial \Omega_{i}$, for $e=s, p$, for $B=D, N$ and for $i=1,2, f$. We will denote the exterior pointing, unit, normal, vector field on $\partial \Omega_{i}$ by $\mathbf{n}_{i}, i=1,2, f$.

Now to rewrite both the pressure problem (11)-(12) and the saturation problem (15)-(16) in $\Omega$ in a multidomain formulation as subdomain problems with nonlinear transmission conditions between different rock types we need to give tranmission conditions on $\gamma_{1}$ and $\gamma_{2}$; cf. [5, 14].

For the pressure equation, to conserve mass, we require continuity of the normal component of the total Darcy velocity $\mathbf{u}$ across interfaces between two rock types. However, we do not have continuity of the global "pressure" $p$ but instead continuity of the phase pressures which in light of equation (4), respectively equation (9), implies continuity of the capillary pressure $\pi$, respectively continuity of $p-\beta$. 
The pressure problem can now be written as

$$
\begin{aligned}
& \nabla \cdot \mathbf{u}_{i}=q_{i}, \quad \mathbf{u}_{i}=-\mathbf{K}_{i} k_{i}\left(s_{i}\right)\left(\nabla p_{i}-\rho_{i}\left(s_{i}\right) \mathbf{u}_{G}\right), \quad \text { in } \Omega_{i}, \quad i=1,2, f, \\
& \mathbf{u}_{i} \cdot \mathbf{n}_{i}=0, \quad \text { on } \Gamma_{i}^{p N}, i=1,2, f, \\
& p_{i}=\bar{p}_{D}, \quad \text { on } \Gamma_{i}^{p D}, i=1,2, f, \\
& \mathbf{u}_{i} \cdot \mathbf{n}_{i}=(-1)^{i+1} \mathbf{u}_{f} \cdot \mathbf{n}_{f}, \quad p_{i}-\beta_{i}\left(s_{i}\right)=p_{f}-\beta_{f}\left(s_{f}\right), \quad \text { on } \gamma_{i}, \quad i=1,2,
\end{aligned}
$$

where $\bar{p}_{D}$ is the Dirichlet boundary data for the global pressure. We point out that (20) implies that the global pressure $p$ is not in general continuous across interfaces between regions with different capillary pressure curves.

For the transmission conditions for the saturation equation, as for the pressure equation, conservation of mass requires that the normal component of the Darcy velocity, this time of the wetting-phase be continuous across the interfaces $\gamma_{i}, i=1,2$. However instead of continuity of the wetting-phase saturation $s$ we have continuity of the capillary pressure $\pi$. The saturations on the two sides of an interface must be such that the capillary pressure is the same on both sides. Note however that since the two capillary pressure curves are different the same capillary pressure value will in general correspond to different saturation values on the two sides of the interface; see Figure 2. The saturation problem when written in the multi domain formulation is as follows:

$$
\begin{array}{ll}
\Phi_{i} \frac{\partial s_{i}}{\partial t}+\nabla \cdot \mathbf{u}_{w i}=q_{w i}, \quad \mathbf{u}_{w i}=\mathbf{r}_{i}+\mathbf{f}_{i}, & \text { in } \Omega_{i}, \quad i=1,2, f, \\
\mathbf{r}_{i}=-\mathbf{K}_{i} \nabla \alpha_{i}\left(s_{i}\right), \quad \mathbf{f}_{i}=f_{w i}\left(s_{i}\right)\left(\mathbf{u}_{i}+f_{G w i}\left(s_{i}\right) \mathbf{K}_{i} \mathbf{u}_{G}\right), & \text { in } \Omega_{i}, \quad i=1,2, f, \\
\mathbf{u}_{w i} \cdot \mathbf{n}_{i}=0, & \text { on } \Gamma_{i}^{s N}, i=1,2, f, \\
s_{i}=\bar{s}_{D}, & \text { on } \Gamma_{i}^{s D}, i=1,2, f, \\
\mathbf{u}_{w i} \cdot \mathbf{n}_{i}=(-1)^{i+1} \mathbf{u}_{w f} \cdot \mathbf{n}_{f}, \quad \pi_{i}\left(s_{i}\right)=\pi_{f}\left(s_{f}\right), & \text { on } \gamma_{i}, \quad i=1,2,
\end{array}
$$

where $\bar{s}_{D}$ is the Dirichlet boundary data for the saturation.

To complete the formulation, we add the initial condition for saturation

$$
s_{i}=s_{i}^{0} \quad \text { in } \Omega_{i}, \quad i=1,2, f .
$$

\section{A reduced model for two-phase flow in a domain with a fracture}




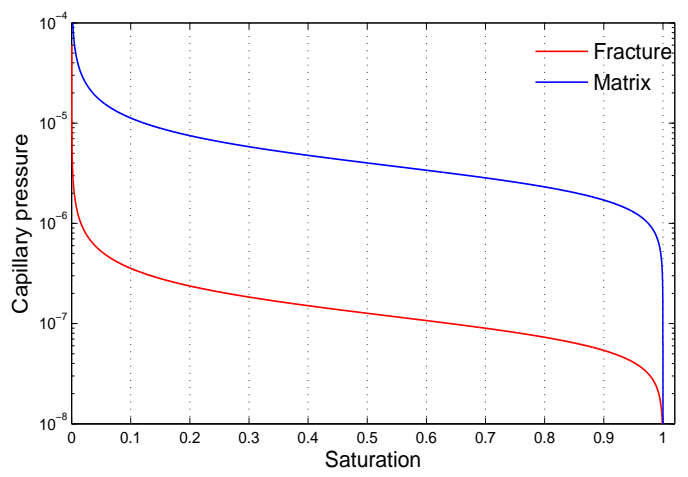

Figure 2: Capillary pressure curves

As in the reduced model of [32] the fracture is represented by a surface $\gamma$ of dimension $(n-1)$ as shown in Figure 3. The idea for the reduced model is to collapse the fracture domain $\Omega_{f}$ onto its central axis $\gamma$, (translating the domains $\Omega_{1}$ and $\Omega_{2}$ by a distance $d / 2$ toward the central axis) and to obtain equations on the resulting interface $\gamma$ by integrating the equations on $\Omega_{f}$ over its transversal cross sections. Before integrating over the cross sections of $\gamma$ however, we decompose $\mathbf{u}_{f}$ into its components normal to and tangential to the fracture $\mathbf{u}_{f}=\mathbf{u}_{f \tau}+\mathbf{u}_{f \mathbf{n}}$ with $\mathbf{u}_{f \mathbf{n}}=\left(\mathbf{u}_{f} \cdot \mathbf{n}\right) \mathbf{n}$ and $\mathbf{u}_{f \tau}=\mathbf{u}_{f}-\mathbf{u}_{f \mathbf{n}}$. Also, we introduce the symbols $\nabla_{\tau}$ and $\nabla_{\mathbf{n}}$ for the tangential and the normal components of the gradient operator. Next, the functions characterizing the fracture medium $\Omega_{f}$, i.e. the porosity, the absolute permeability and the depth, are averaged over cross sections of $\Omega_{f}$ normal to the direction of the fracture or simply assumed to be invariant in the normal direction. We assume that the absolute permeability tensor $\mathbf{K}_{f}$ can be written as the sum of a normal part $\mathbf{K}_{f \mathbf{n}}$ and a tangential part $\mathbf{K}_{f \tau}$, and for simplicity we assume that these are both invariant in the normal direction. The resulting model consists of a two-phase flow problem in the three-dimensional subdomains coupled with a lower dimensional two-phase flow problem in the interface of co-dimension 1.

\subsection{The pressure equation in the fracture for the reduced model}

The total mass conservation in equation (17) in $\Omega_{f}$ may be written in the reference system $\{\mathbf{n}, \boldsymbol{\tau}\}$ as follows:

$$
\nabla_{\tau} \cdot \mathbf{u}_{f}+\nabla_{\mathbf{n}} \cdot \mathbf{u}_{f}=q_{f} \quad \text { in } \Omega_{f}
$$




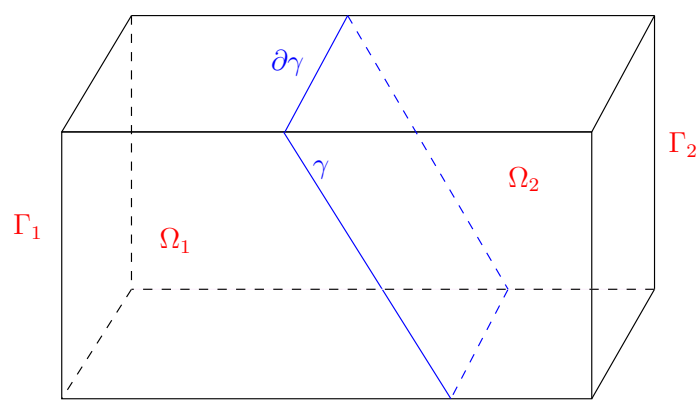

Figure 3: The domain $\Omega$ with the fracture interface $\gamma$.

Integrating this equation across the cross-sections of the fracture results in a mass balance equation in two dimensions with an additional source term representing the difference between what flows into the fracture from the subdomains $\Omega_{i}, i=1,2$, and what flows out of the fracture toward the subdomains:

$$
\nabla_{\tau} \cdot \mathbf{u}_{\gamma}=q_{\gamma}+\left(\mathbf{u}_{1} \cdot \mathbf{n}_{1}+\mathbf{u}_{2} \cdot \mathbf{n}_{2}\right) \quad \text { in } \gamma
$$

where $\mathbf{u}_{\gamma}$, the total Darcy velocity in $\gamma$, and $q_{\gamma}$ are defined by

$$
\mathbf{u}_{\gamma}=\int_{-d / 2}^{d / 2} \mathbf{u}_{f \tau} d n, \quad q_{\gamma}=\int_{-d / 2}^{d / 2} q_{f} d n .
$$

To obtain the expression for the additional source term we have used the continuity of the total Darcy velocity at the interfaces $\gamma_{1}$ and $\gamma_{2}$ to replace $-\left.\mathbf{u}_{f} \cdot \mathbf{n}_{f}\right|_{\gamma_{i}}$ by $\mathbf{u}_{i} \cdot \mathbf{n}_{i}, i=1,2$.

Writing Darcy's law in equation (17) in $\Omega_{f}$ with the reference system $\{\boldsymbol{\tau}, \mathbf{n}\}$ and for simplicity neglecting inside the fracture the component of the gravity term normal to the fracture yields

$$
\begin{array}{ll}
\mathbf{u}_{f \tau}=-\mathbf{K}_{f \tau} k_{f}\left(s_{f}\right)\left(\nabla_{\tau} p_{f}-\rho_{f}\left(s_{f}\right) \mathbf{u}_{G, \tau}\right) & \text { in } \Omega_{f}, \\
\mathbf{u}_{f \mathbf{n}}=-\mathbf{K}_{f \mathbf{n}} k_{f}\left(s_{f}\right) \nabla_{\mathbf{n}} p_{f} & \text { in } \Omega_{f},
\end{array}
$$

where with an abuse of notation we have written $\mathbf{K}_{f \mathbf{n}}$ for the single non zero component of the tensor $\mathbf{K}_{f \mathbf{n}}$ and we kept the vector notation for the one-dimensional vector $\mathbf{u}_{f \mathbf{n}}$. We also introduced the tangential component of the gravity vector, $\mathbf{u}_{G}=\mathbf{u}_{G, \tau}+\mathbf{u}_{G, \mathbf{n}}$. Writing $\mathbf{K}_{\gamma}, k_{\gamma}$ and $\rho_{\gamma}$ for $d \mathbf{K}_{f \tau}, k_{f}$ and $\rho_{f}$ respectively and integrating equation (29) across the cross-sections of the fracture we obtain

$$
\mathbf{u}_{\gamma}=-\mathbf{K}_{\gamma} k_{\gamma}\left(s_{\gamma}\right)\left(\nabla_{\tau} p_{\gamma}-\rho_{\gamma}\left(s_{\gamma}\right) \mathbf{u}_{G, \tau}\right) \quad \text { in } \gamma
$$


where

$$
p_{\gamma}=\frac{1}{d} \int_{-d / 2}^{d / 2} p_{f} d n, \quad s_{\gamma}=\frac{1}{d} \int_{-d / 2}^{d / 2} s_{f} d n .
$$

Thus the pressure equation in the interface fracture consists of (28) together with (31). This equation takes into account the exchanges with the subdomains through the additional source term of (28). Equation (30) will be used to give a boundary condition on $\gamma$ for the pressure equation in the subdomain $\Omega_{i}, i=1,2$, and thus to allow the pressure equations in the subdomains to take into account exchange with the fracture. Integrating this time over the half-cross-sections of the fracture and using the approximations

$$
\left.\int_{-d / 2}^{0} \mathbf{u}_{f \mathbf{n}} d n \approx \frac{d}{2}\left(\mathbf{u}_{f} \cdot \mathbf{n}\right)\right|_{\gamma_{1}} \text { and }\left.\int_{0}^{d / 2} \mathbf{u}_{f \mathbf{n}} d n \approx \frac{d}{2}\left(\mathbf{u}_{f} \cdot \mathbf{n}\right)\right|_{\gamma_{2}}
$$

we obtain two equations, one for each $i, i=1,2$ :

$$
\begin{aligned}
& \left.\frac{d}{2}\left(\mathbf{u}_{1} \cdot \mathbf{n}\right)\right|_{\gamma_{1}}=-\mathbf{K}_{f \mathbf{n}} k_{\gamma}\left(s_{\gamma}\right)\left(p_{\gamma}-\left.p_{f}\right|_{\gamma_{1}}\right), \\
& \left.\frac{d}{2}\left(\mathbf{u}_{2} \cdot \mathbf{n}\right)\right|_{\gamma_{2}}=-\mathbf{K}_{f \mathbf{n}} k_{\gamma}\left(s_{\gamma}\right)\left(\left.p_{f}\right|_{\gamma_{2}}-p_{\gamma}\right),
\end{aligned}
$$

where we have again used the continuity of the normal component of the total Darcy velocity across $\gamma_{i}$. Using the continuity of $p-\beta(s)$ across $\gamma_{i}$ we obtain

$$
\begin{aligned}
& \left.\frac{d}{2}\left(\mathbf{u}_{1} \cdot \mathbf{n}\right)\right|_{\gamma_{1}}=-\mathbf{K}_{f \mathbf{n}} k_{\gamma}\left(s_{\gamma}\right)\left(\left(p_{\gamma}-\beta_{\gamma}\left(s_{\gamma}\right)\right)-\left(p_{1}-\beta_{1}\left(s_{1}\right)\right),\right. \\
& \left.\frac{d}{2}\left(\mathbf{u}_{2} \cdot \mathbf{n}\right)\right|_{\gamma_{2}}=-\mathbf{K}_{f \mathbf{n}} k_{\gamma}\left(s_{\gamma}\right)\left(\left(p_{2}-\beta_{2}\left(s_{2}\right)-\left(p_{\gamma}-\beta_{\gamma}\left(s_{\gamma}\right)\right)\right),\right.
\end{aligned}
$$

or with the notation

$$
\begin{gathered}
\kappa\left(s_{\gamma}\right)=-\frac{\mathbf{K}_{f \mathbf{n}} k_{\gamma}\left(s_{\gamma}\right)}{d / 2} \\
\mathbf{u}_{i} \cdot \mathbf{n}_{i}+\kappa\left(s_{\gamma}\right)\left(p_{i}-\beta_{i}\left(s_{i}\right)\right)=\kappa\left(s_{\gamma}\right)\left(p_{\gamma}-\beta_{\gamma}\left(s_{\gamma}\right)\right), i=1,2 .
\end{gathered}
$$

\subsection{The saturation equation in the fracture for the reduced model}

We proceed for the saturation equation (21),(22) in the same manner as we did for the pressure equation. We integrate their tangential components for $i=f$ over cross-sections in the direction normal to the fracture, and using the continuity of the wetting-phase flux across $\gamma_{1}$ and 
$\gamma_{2}$ we obtain

$$
\begin{aligned}
& \Phi_{\gamma} \frac{\partial s_{\gamma}}{\partial t}+\nabla_{\tau} \cdot \mathbf{u}_{w \gamma}=q_{w \gamma}+\left(\mathbf{u}_{w 1} \cdot \mathbf{n}_{1}+\mathbf{u}_{w 2} \cdot \mathbf{n}_{2}\right), \quad \text { in } \gamma, \\
& \mathbf{u}_{w \gamma}=\mathbf{r}_{\gamma}+\mathbf{f}_{\gamma} \quad \text { in } \gamma, \\
& \mathbf{r}_{\gamma}=-\mathbf{K}_{\gamma} \nabla_{\tau} \alpha_{\gamma}\left(s_{\gamma}\right), \quad \mathbf{f}_{\gamma}=f_{w \gamma}\left(s_{\gamma}\right)\left(\mathbf{u}_{\gamma}+f_{G w \gamma}\left(s_{\gamma}\right) \mathbf{K}_{\gamma} \mathbf{u}_{G, \tau}\right), \quad \text { in } \gamma,
\end{aligned}
$$

where we introduced

$$
\mathbf{u}_{w \gamma}=\int_{-d / 2}^{d / 2} \mathbf{u}_{w f \tau} d n, \quad q_{w \gamma}=\int_{-d / 2}^{d / 2} q_{w f \tau} d n, \quad \mathbf{r}_{\gamma}=\int_{-d / 2}^{d / 2} \mathbf{r}_{f \tau} d n \quad \text { and } \quad \mathbf{f}_{\gamma}=\int_{-d / 2}^{d / 2} \mathbf{f}_{f \tau} d n,
$$

and where we have written $\Phi_{\gamma}, \alpha_{\gamma}, f_{\gamma}$ and $f_{G w \gamma}$ for $\Phi_{f}, \alpha_{f}, f_{f}$ and $d f_{G w f}$, respectively.

Thus with (33), (34) and (35) making up the saturation equation along $\gamma$, to obtain a boundary condition on $\gamma$ for the saturation equation in $\Omega_{1}$ and that in $\Omega_{2}$, just as we did for the pressure equation for the reduced model, we turn to the normal component of Darcy's law, but this time for that of the wetting-phase. Since we have neglected gravity effects across the fracture in the normal direction $\mathbf{n}$, we have

$$
\mathbf{u}_{w f \mathbf{n}}=-\mathbf{K}_{f \mathbf{n}} k_{w f}\left(s_{f}\right) \nabla_{\mathbf{n}} p_{f w} .
$$

Integrating this equation across the normal half-cross-sections of the fracture and using the approximations

$$
\left.\int_{-d / 2}^{0} \mathbf{u}_{w f \mathbf{n}} d n \approx \frac{d}{2}\left(\mathbf{u}_{w 1} \cdot \mathbf{n}\right)\right|_{\gamma_{1}} \text { and }\left.\int_{0}^{d / 2} \mathbf{u}_{w f \mathbf{n}} d n \approx \frac{d}{2}\left(\mathbf{u}_{w 2} \cdot \mathbf{n}\right)\right|_{\gamma_{2}}
$$

we obtain

$$
\left.\frac{d}{2} \mathbf{u}_{w 1} \cdot \mathbf{n}\right|_{\gamma_{1}}=-\mathbf{K}_{f \mathbf{n}} k_{w f}\left(s_{f}\right)\left(p_{\gamma w}-\left.p_{f w}\right|_{\gamma_{1}}\right),\left.\quad \frac{d}{2} \mathbf{u}_{w 2} \cdot \mathbf{n}\right|_{\gamma_{2}}=-\mathbf{K}_{f \mathbf{n}} k_{w f}\left(s_{f}\right)\left(\left.p_{f w}\right|_{\gamma_{2}}-p_{\gamma w}\right),
$$

where we have used the continuity of the normal component of the wetting phase velocity across $\gamma_{i}, i=1,2$.

Now, using equation (10) and the continuity of the capillary pressure and that of the term $p-\beta$ across $\gamma_{i}$ we obtain

$$
\mathbf{u}_{w i} \cdot \mathbf{n}_{i}+\kappa_{w}\left(s_{\gamma}\right)\left(p_{i}-\beta_{i}-\frac{1}{2} \pi_{i}\right)=\kappa_{w}\left(s_{\gamma}\right)\left(p_{\gamma}-\beta_{\gamma}-\frac{1}{2} \pi_{\gamma}\right),
$$

where $\kappa_{w}\left(s_{\gamma}\right)=-\frac{\mathbf{K}_{f \mathbf{n}} k_{w \gamma}\left(s_{f}\right)}{d / 2}$. Using the pressure transmission condition (32), we can rewrite these equations as, for $i=1,2$,

$$
\mathbf{u}_{w i} \cdot \mathbf{n}_{i}-\kappa_{w}\left(s_{\gamma}\right) \frac{\pi_{i}\left(s_{i}\right)}{2}=-\kappa_{w}\left(s_{\gamma}\right) \frac{\pi_{\gamma}\left(s_{\gamma}\right)}{2}+f_{w \gamma}\left(s_{\gamma}\right) \mathbf{u}_{i} \cdot \mathbf{n}_{i}
$$


since $\frac{\kappa_{w}\left(s_{\gamma}\right)}{\kappa\left(s_{\gamma}\right)}=f_{w \gamma}\left(s_{\gamma}\right)$.

\subsection{The reduced model}

Now we collect all of the equations for the reduced model, mass conservation, Darcy's law, transmission conditions, and boundary conditions. The pressure equations in the matrix subdomains are

$$
\begin{array}{ll}
\nabla \cdot \mathbf{u}_{i}=q_{i}, \quad \mathbf{u}_{i}=-\mathbf{K}_{i} k_{i}\left(s_{i}\right)\left(\nabla p_{i}-\rho_{i}\left(s_{i}\right) \mathbf{u}_{G}\right), & \text { in } \Omega_{i}, \\
\mathbf{u}_{i} \cdot \mathbf{n}_{i}+\kappa\left(s_{\gamma}\right) p_{i}=\kappa\left(s_{\gamma}\right)\left(p_{\gamma}-\beta_{\gamma}\left(s_{\gamma}\right)+\beta_{i}\left(s_{i}\right)\right), & \text { on } \gamma, \\
\mathbf{u}_{i} \cdot \mathbf{n}_{i}=0, & \text { on } \Gamma_{i}^{p N}, \\
p_{i}=\bar{p}_{D}, & \text { on } \Gamma_{i}^{p D},
\end{array}
$$

coupled with the pressure equations in the fracture interface

$$
\begin{aligned}
& \nabla_{\tau} \cdot \mathbf{u}_{\gamma}=q_{\gamma}+\mathbf{u}_{1} \cdot \mathbf{n}_{1}+\mathbf{u}_{2} \cdot \mathbf{n}_{2}, \quad \mathbf{u}_{\gamma}=-\mathbf{K}_{\gamma} k_{\gamma}\left(s_{\gamma}\right)\left(\nabla_{\tau} p_{\gamma}-\rho_{\gamma}\left(s_{\gamma}\right) \mathbf{u}_{G, \tau}\right), \quad \text { in } \gamma, \\
& p_{\gamma}=\bar{p}_{D}, \text { on } \partial \gamma^{p D}, \quad \mathbf{u}_{\gamma}=0, \text { on } \partial \gamma^{p N} .
\end{aligned}
$$

where $\partial \gamma=\partial \gamma^{p N} \cup \partial \gamma^{p N}$ is divided into its Dirichlet and Neumann parts for the pressure equation.

The saturation equations in the matrix subdomains are

$$
\begin{array}{ll}
\Phi_{i} \frac{\partial s_{i}}{\partial t}+\nabla \cdot \mathbf{u}_{w i}=q_{i}, \quad \mathbf{u}_{w i}=\mathbf{r}_{i}+\mathbf{f}_{i}, & \text { in } \Omega_{i}, \\
\mathbf{r}_{i}=-\mathbf{K}_{i} \nabla \alpha_{i}\left(s_{i}\right), \quad \mathbf{f}_{i}\left(s_{i}\right)=f_{w i}\left(s_{i}\right)\left(\mathbf{u}_{i}+f_{G w i}\left(s_{i}\right) \mathbf{K}_{i} \mathbf{u}_{G}\right), & \text { in } \Omega_{i}, \\
\mathbf{u}_{w i} \cdot \mathbf{n}_{i}-\kappa_{w}\left(s_{\gamma}\right) \frac{\pi_{i}\left(s_{i}\right)}{2}=-\kappa_{w}\left(s_{\gamma}\right) \frac{\pi_{\gamma}\left(s_{\gamma}\right)}{2}+f_{w \gamma}\left(s_{\gamma}\right) \mathbf{u}_{i} \cdot \mathbf{n}_{i}, & \text { on } \gamma, \\
\mathbf{u}_{w i} \cdot \mathbf{n}_{i}=0, & \text { on } \Gamma_{i}^{s N}, \\
s_{i}=\bar{s}_{D}, & \text { on } \Gamma_{i}^{s D},
\end{array}
$$

coupled with the saturation equations in the fracture interface

$$
\begin{aligned}
& \Phi_{\gamma} \frac{\partial s_{\gamma}}{\partial t}+\nabla_{\tau} \cdot \mathbf{u}_{w \gamma}=q_{w \gamma}+\mathbf{u}_{w 1} \cdot \mathbf{n}_{1}+\mathbf{u}_{w 2} \cdot \mathbf{n}_{2}, \quad \mathbf{u}_{w \gamma}=\mathbf{r}_{\gamma}+\mathbf{f}_{\gamma} \quad \text { in } \gamma, \\
& \mathbf{r}_{\gamma}=-\mathbf{K}_{\gamma} \nabla_{\tau} \alpha_{f}\left(s_{\gamma}\right), \quad \mathbf{f}_{\gamma}\left(s_{\gamma}\right)=f_{w \gamma}\left(s_{\gamma}\right)\left(\mathbf{u}_{\gamma}+f_{G w \gamma}\left(s_{\gamma}\right) \mathbf{K}_{\gamma} \mathbf{u}_{G, \tau}\right) \quad \text { in } \gamma, \\
& s_{\gamma}=\bar{s}_{D} \text {, on } \partial \gamma^{s D}, \quad \mathbf{u}_{w \gamma}=0 \text {, on } \partial \gamma^{s N} \text {. }
\end{aligned}
$$

where $\partial \gamma=\partial \gamma^{p N} \cup \partial \gamma^{p N}$ is divided into its Dirichlet and Neumann parts for the saturation equation. 
Again we point out that the equations in the interface receive input from those in the subdomains through the additional source terms in the conservation equations (41) and (48), which represent the contributions of the subdomains to the flow in the fracture, while equations in the subdomains receive input from those in the fracture interface through the transmission conditions (38) and (45) imposed on $\gamma$ which provide boundary conditions of Robin type to the subdomain solvers.

We note that for the reduced model, the capillary pressure, and even the phase pressures, may be discontinuous across the fracture interface $\gamma$ as $s_{\gamma}$ does not represent the saturation value on either boundary $\gamma_{i}$ of the full fracture $\Omega_{f}$ but an average value across the cross-sections of $\Omega_{f}$.

We also point out that the reduced model is valid for fractures with permeability higher than that of the rock matrix as well as for fractures which act as barriers, and with this observation it is interesting to consider the two limit cases which occur when the coefficients in the transmission conditions of (38) and (45) are very large and when they are very small:

- If $\mathbf{K}_{f \mathbf{n}} \longrightarrow \infty$, then $\kappa\left(s_{\gamma}\right) \longrightarrow \infty$ and $\kappa_{w}\left(s_{\gamma}\right) \longrightarrow \infty$ and dividing Equation (32) by $\kappa\left(s_{\gamma}\right)$ and Equation (36) by $\kappa_{w}\left(s_{\gamma}\right)$ we obtain for $i=1,2$

$$
p_{i}-\beta_{i}\left(s_{i}\right)=p_{\gamma}-\beta_{\gamma}\left(s_{\gamma}\right), \quad \pi_{i}\left(s_{i}\right)=\pi_{\gamma}\left(s_{\gamma}\right) .
$$

The phase pressure and capillary pressure discontinuities across $\gamma$ decrease when the absolute permeability in the fracture increases in comparison to the matrix permeability.

- When neglecting capillary pressure the transmission conditions (32), (36) become

$$
\mathbf{u}_{i} \cdot \mathbf{n}_{i}+\kappa\left(s_{\gamma}\right) p_{i}=\kappa_{\gamma}\left(s_{\gamma}\right) p_{\gamma}, \quad \mathbf{u}_{w i} \cdot \mathbf{n}_{i}=f_{\gamma}\left(s_{\gamma}\right) \mathbf{u}_{i} \cdot \mathbf{n}_{i} .
$$

\section{Intersecting fractures}

In this section we consider a simple model in which the domain $\Omega$ is subdivided into 3 subdomains $\Omega_{i}, i \in I=\{1,2,3\}$ separated by intersecting fracture interfaces $\gamma_{i j}=\partial \Omega_{i} \cap$ $\partial \Omega_{j},(i, j) \in S=\{(1,2),(2,3),(3,1)\}$ as in Fig. 4. Again for simplicity, we assume that each of the fracture interfaces is planar and that $T=\gamma_{i j} \cap \gamma_{j k} \subset \Omega$ whenever $(i, j)$ and $(j, k)$ belong to $S$. We set

$$
\Gamma_{i}=\partial \Omega \cap \partial \Omega_{i}, \quad \gamma=\underset{(i, j) \in S}{\cup} \gamma_{i j}, \quad \gamma_{i}=\partial \Omega_{i} \cap \gamma=\underset{(j, k) \in S}{\cup} \gamma_{j k}
$$




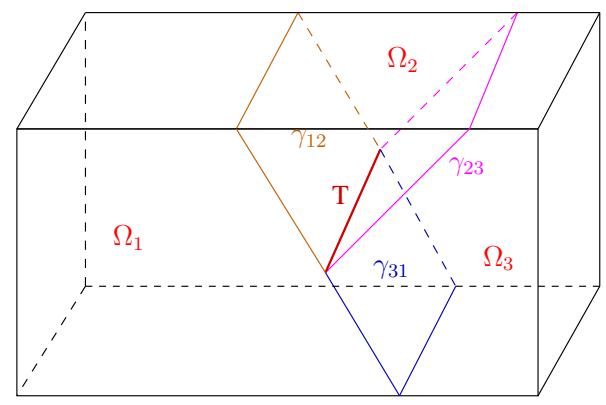

Figure 4: A domain $\Omega$ with intersecting fractures

As earlier, $\mathbf{n}_{i}$ is the exterior unit normal vector field on $\partial \Omega_{i}$ and $\mathbf{n}_{i j}$ is the exterior unit normal vector field on $\partial \gamma_{i j}$ (in the plane of $\gamma_{i j}$ ).

Concerning $T$ where the interfaces intersect we assume that there is no one-dimensional flow along $T$. If we assume also that the fractures have all the same high permeability compared to the matrix rock we can assume continuity of phase pressure and with mass conservation we have at the intersection

$$
\begin{array}{ll}
\sum_{(i, j) \in S} \mathbf{u}_{w i j} \cdot \mathbf{n}_{i j}=0, & \text { on } T, \\
\pi_{i j}\left(s_{i j}\right)=\pi_{k l}\left(s_{k l}\right), \quad(i, j),(k, l) \in S,(i, j) \neq(k, l), & \text { on } T, \\
\sum_{(i, j) \in S} \mathbf{u}_{i j} \cdot \mathbf{n}_{i j}=0, & \text { on } T, \\
p_{i j}-\beta_{i j}\left(s_{i j}\right)=p_{k l}-\beta_{k l}\left(s_{k l}\right), \quad(i, j),(k, l) \in S,(i, j) \neq(k, l), & \text { on } T .
\end{array}
$$

In this model continuity of phase pressures as well as of phase fluxes are imposed at fracture intersections since we assumed that the fractures have a high permeability compared with that in the matrix rock. In other situations, like for instance the intersection of a barrier with a high permeability fracture, other conditions based on physical assumptions must be imposed. Thus in our numerical experiment described in section 9.3 we assumed that the barrier does not cut through the high permeability fractures and that there is no flow entering the barrier at the fracture intersection.

Remark 1. If we would like to consider a flow along $T$, we would have to add one-dimensional pressure and saturation equations with source terms representing the exchange of fluid between 
the fractures $\gamma_{i j}$ with continuity of phase pressures and capillary pressures between $T$ and the fractures $\gamma_{i j}$.

\section{Multidomain formulation}

Since the fractures are represented as interfaces between subdomains of the porous medium it is natural to apply domain decomposition methods. The domain decomposition method that we present here is an extension to two-phase flow of that used for single-phase flow in [37]. The unknowns from each subdomain are eliminated and the problem is formulated as a problem set on the interface $\gamma$. This formulation is very useful in order to use different time steps in the subdomains and in the fracture [27, 29] even though for simplicity of notation, we will use in this article equal time steps all over the domain. Also, for simplicity, throughout this section, all problems are written in strong form, we suppose that homogeneous Dirichlet boundary conditions are imposed everywhere on $\partial \Omega$ and we consider the same situation as in the previous section.

\subsection{Pressure equation}

For each subdomain $\Omega_{i}, i=1,2.3$ we introduce the space $M^{\gamma_{i}}=L^{2}\left(\gamma_{i}\right)$, and the linear Robin to Neumann operator $\mathcal{L}_{p i}^{R t N}$ defined by

$$
\begin{aligned}
\mathcal{L}_{p i}^{R t N}: & L^{2}\left(0, T_{f} ; M^{\gamma_{i}}\right) \longrightarrow L^{2}\left(0, T_{f} ; M^{\gamma_{i}}\right) \\
\lambda_{i} & \longmapsto-\left.\left(\mathbf{u}_{i} \cdot \mathbf{n}_{i}\right)\right|_{\gamma_{i}}
\end{aligned}
$$

with $\left(\mathbf{u}_{i}, p_{i}\right)$ the solution of the pressure problem

$$
\begin{array}{ll}
\nabla \cdot \mathbf{u}_{i}=q_{i}, \quad \mathbf{u}_{i}=-\mathbf{K}_{i} k_{i}\left(s_{i}\right)\left(\nabla p_{i}-\rho_{i}\left(s_{i}\right) \mathbf{u}_{G}\right), & \text { in } \Omega_{i}, \\
\mathbf{u}_{i} \cdot \mathbf{n}_{i}+\kappa\left(s_{i}\right) p_{i}=\kappa\left(s_{i}\right)\left(\lambda_{i}-\beta_{\gamma}\left(s_{\gamma}\right)+\beta_{i}\left(s_{i}\right)\right), & \text { on } \gamma_{i}, \\
p_{i}=0, & \text { on } \Gamma_{i} .
\end{array}
$$

Obviously the operator $\mathcal{L}_{p i}^{R t N}$ depends on $s_{i}$ but this dependence is not shown in order to make the notation less cumbersome.

Then, by extending $\mathcal{L}_{p i}^{R t N}\left(\lambda_{i}\right)$ to all of $\gamma$ with an extension by 0 on $\gamma \backslash \gamma_{i}$, we can define

$$
\mathcal{L}_{p}^{R t N}\left(\lambda_{\gamma}\right)=\sum_{i \in I} \mathcal{L}_{p i}^{R t N}\left(\lambda_{i}\right)
$$


Also at the intersection $T$ between the fractures, we define the following operator

$$
\begin{aligned}
\mathcal{L}_{p T}^{R t N}\left(\lambda_{T}\right): & L^{2}\left(0, T_{f} ; L^{2}(T)\right) \longrightarrow L^{2}\left(0, T_{f} ; L^{2}(T)\right), \\
& \lambda_{T} \longmapsto-\left.\sum_{(i, j) \in S}\left(\mathbf{u}_{i j} \cdot \mathbf{n}_{i j}\right)\right|_{T},
\end{aligned}
$$

where $\mathbf{u}_{i j}=\left.\mathbf{u}_{\gamma}\right|_{\gamma_{i j}}$ with $\left(\mathbf{u}_{\gamma}, \lambda_{\gamma}\right)$ the solution of

$$
\begin{array}{ll}
\nabla_{\tau} \cdot \mathbf{u}_{\gamma}+\mathcal{L}_{p}^{R t N}\left(\lambda_{\gamma}\right)=q_{\gamma}, \quad \mathbf{u}_{\gamma}=-\mathbf{K}_{\gamma} k_{\gamma}\left(s_{\gamma}\right)\left(\nabla_{\tau} \lambda_{\gamma}-\rho_{\gamma}\left(s_{\gamma}\right) \mathbf{u}_{G, \tau}\right), & \text { in } \gamma, \\
\lambda_{\gamma}=0, & \text { on } \partial \gamma, \\
\lambda_{\gamma}=\lambda_{T}+\beta_{\gamma}\left(s_{\gamma}\right), & \text { on } T .
\end{array}
$$

Then the interface formulation of the pressure equation is to find $\left(\mathbf{u}_{\gamma}, p_{\gamma}, p_{T}\right)$ solution of

$$
\begin{array}{ll}
\nabla_{\tau} \cdot \mathbf{u}_{\gamma}+\mathcal{L}_{p}^{R t N}\left(p_{\gamma}\right)=q_{\gamma}, \quad \mathbf{u}_{\gamma}=-\mathbf{K}_{\gamma} k_{\gamma}\left(s_{\gamma}\right)\left(\nabla_{\tau} p_{\gamma}-\rho_{\gamma}\left(s_{\gamma}\right) \mathbf{u}_{G, \tau}\right), & \text { in } \gamma, \\
p_{\gamma}=0, & \text { on } \partial \gamma, \\
p_{T}=p_{i j}-\beta_{i j}\left(s_{i j}\right),(i, j) \in S, \quad \mathcal{L}_{p T}^{R t N}\left(p_{T}\right)=0, & \text { on } T .
\end{array}
$$

\subsection{Saturation equation}

Similarly we define the nonlinear Robin to Neumann operator:

$$
\begin{aligned}
\mathcal{L}_{s i}^{R t N}: & L^{2}\left(0, T_{f} ; M^{\gamma_{i}}\right) \longrightarrow L^{2}\left(0, T_{f} ; M^{\gamma_{i}}\right) \\
\theta_{i} & \longmapsto-\left.\left(\mathbf{u}_{w i} \cdot \mathbf{n}_{i}\right)\right|_{\gamma_{i}}
\end{aligned}
$$

where $\left(\mathbf{u}_{w i}, s_{i}\right)$ is the solution of

$$
\begin{array}{ll}
\Phi_{i} \frac{\partial s_{i}}{\partial t}+\nabla \cdot \mathbf{u}_{w i}=q_{i}, \quad \mathbf{u}_{w i}=\mathbf{r}_{i}+\mathbf{f}_{i}, & \text { in } \Omega_{i}, \\
\mathbf{r}_{i}=-\mathbf{K}_{i} \nabla \alpha_{i}\left(s_{i}\right), \quad \mathbf{f}_{i}=f_{w i}\left(s_{i}\right)\left(\mathbf{u}_{i}+f_{G w i}\left(s_{i}\right) \mathbf{u}_{G}\right), & \text { in } \Omega_{i}, \\
\mathbf{u}_{w i} \cdot \mathbf{n}_{i}-\kappa_{w}\left(\theta_{i j}\right) \frac{\pi_{i}\left(s_{i}\right)}{2}=-\kappa_{w}\left(\theta_{i}\right) \frac{\pi_{\gamma}\left(\theta_{i}\right)}{2}+f_{w \gamma}\left(\theta_{i}\right) \mathbf{u}_{i} \cdot \mathbf{n}_{i}, & \text { on } \gamma_{i}, \\
s_{i}=0, & \text { on } \Gamma_{i} .
\end{array}
$$

For simplicity of notation, we do not show the dependence of the operator $\mathcal{L}_{s i}^{R t N}$ on $p_{i}$.

We extend $\mathcal{L}_{s i}^{R t N}\left(\theta_{i}\right)$ to all of $\gamma$ with an extension by 0 on $\gamma \backslash \gamma_{i}$, and we define

$$
\mathcal{L}_{s}^{R t N}\left(\theta_{\gamma}\right)=\sum_{\substack{i \in I \\ 16}} \mathcal{L}_{s i}^{R t N}\left(\theta_{i}\right)
$$


Also at the intersection $T$ between the fractures, we define the following operator

$$
\begin{aligned}
& \mathcal{L}_{s T}^{R t N}\left(\pi_{T}\right): L^{2}\left(0, T_{f} ; L^{2}(T)\right) \longrightarrow L^{2}\left(0, T_{f} ; L^{2}(T)\right), \\
& \pi_{T} \longmapsto-\left.\sum_{(i, j) \in S}\left(\mathbf{u}_{w i j} \cdot \mathbf{n}_{i j}\right)\right|_{T},
\end{aligned}
$$

where $\mathbf{u}_{w i j}=\left.\mathbf{u}_{w \gamma}\right|_{\gamma_{i j}}$ with $\left(\mathbf{u}_{w \gamma}, \theta_{\gamma}\right)$ the solution of

$$
\begin{array}{lr}
\Phi_{\gamma} \frac{\partial \theta_{\gamma}}{\partial t}+\nabla_{\tau} \cdot \mathbf{u}_{w \gamma}+\mathcal{L}_{s}^{R t N}\left(\theta_{\gamma}\right)=q_{w \gamma}, \quad \mathbf{u}_{w \gamma}=\mathbf{r}_{\gamma}+\mathbf{f}_{\gamma}, & \text { in } \gamma, \\
\mathbf{r}_{\gamma}=-\mathbf{K}_{\gamma} \nabla_{\tau} \alpha_{\gamma}\left(\theta_{\gamma}\right), \quad \mathbf{f}_{\gamma}=f_{w \gamma}\left(s_{\gamma}\right)\left(\mathbf{u}_{\gamma}+f_{G w \gamma}\left(s_{\gamma}\right) \mathbf{u}_{G, \tau}\right), & \text { in } \gamma, \\
\theta_{\gamma}=0, & \text { on } \partial \gamma, \\
\pi_{i j}\left(\theta_{\gamma}\right)=\pi_{T}, & \text { on } T .
\end{array}
$$

Then the interface formulation of the saturation equation is to find $\left(\mathbf{u}_{w \gamma}, s_{\gamma}, \pi_{T}\right)$ solution of

$$
\begin{array}{ll}
\Phi_{\gamma} \frac{\partial s_{\gamma}}{\partial t}+\nabla_{\tau} \cdot \mathbf{u}_{w \gamma}+\mathcal{L}_{s}^{R t N}\left(s_{\gamma}\right)=q_{w \gamma} \quad \mathbf{u}_{w \gamma}=\mathbf{r}_{\gamma}+\mathbf{f}_{\gamma}, & \text { in } \gamma, \\
\mathbf{r}_{\gamma}=-\mathbf{K}_{\gamma} \nabla_{\tau} \alpha_{\gamma}\left(s_{\gamma}\right), \quad \mathbf{f}_{\gamma}=f_{w \gamma}\left(s_{\gamma}\right)\left(\mathbf{u}_{\gamma}+f_{G w \gamma}\left(s_{\gamma}\right) \mathbf{u}_{G, \tau}\right), & \text { in } \gamma, \\
s_{\gamma}=0, & \text { on } \partial \gamma, \\
\pi_{\gamma}\left(s_{\gamma}\right)=\pi_{T}, \quad \mathcal{L}_{s T}^{R t N}\left(\pi_{T}\right)=0, & \text { on } T,
\end{array}
$$

\section{Numerical discretization for the interface model}

For the numerical discretization of the pressure equations both in the matrix (37)-(40) and in the fracture (41),(42) we use a mixed finite element method, more specifically the RaviartThomas-Nédélec method of lowest order, in 3 dimensions in the matrix and in 2 dimensions for the fracture. The saturation equation is time dependent and we use a splitting method that permits us to solve a diffusion equation with a backward Euler scheme and an advection equation with a forward Euler scheme. The spatial discretization for the diffusion equation is precisely the same mixed finite element method as is used for the pressure equation. The spatial approximation for the advective equation is a cell-centered finite volume method of first order of Godunov type, again in 3-D for the matrix and in 2-D for the fracture. This diffusion-advection splitting is appropriate for advection dominated problems as in the examples shown in Section 9. 


\subsection{Spatial approximation}

For simplicity, in this section, we suppose that the domain is separated into only two subdomains, $\Omega_{1}$ and $\Omega_{2}$, by a single fracture-interface $\gamma$. Let $\mathcal{T}_{h}^{i}$ denote a finite-element mesh on $\Omega_{i}$ made up of tetrahedra. We suppose that these meshes are such that $\mathcal{T}_{h}=\mathcal{T}_{h}^{1} \cup \mathcal{T}_{h}^{2}$ forms a conforming finite element mesh on all of $\Omega$. Let $\mathcal{F}_{h}$ be the set of all faces of elements of $\mathcal{T}_{h}$. For each $K \in \mathcal{T}_{h}^{i}, i=1$ or 2 , let $\mathcal{F}_{K}$ denote the set of faces of $K$. If $K \in \mathcal{T}_{h}^{i}$ and $L \in \mathcal{T}_{h}^{i}, K \neq L$, share a face $\sigma$, then $\sigma$ will sometimes be denoted $K \mid L$. We will also make use of the following sets of faces:

$$
\mathcal{F}_{h}^{i}=\bigcup_{K \in \mathcal{T}_{h}^{i}} \mathcal{F}_{K}, \quad \mathcal{F}_{h}^{\gamma}=\left\{\sigma \in \mathcal{F}_{h}: \sigma \subset \gamma\right\}, \quad \mathcal{F}_{h}^{\text {ext }}=\left\{\sigma \in \mathcal{F}_{h}: \sigma \subset \partial \Omega\right\} .
$$

Let $\mathcal{T}_{h}^{\gamma}$ be the finite-element mesh on $\gamma$ induced by $\Omega_{i}, i=1,2$; i. e. $\mathcal{T}_{h}^{\gamma}$ is the set of faces of $\mathcal{T}_{h}^{i}$ lying on $\gamma$. We denote by $\mathcal{E}_{h}^{\gamma}$ the set of edges of the triangles in $\mathcal{T}_{h}^{\gamma}$.

The approximate scalar unknowns, pressures $p_{h, i}$ and $p_{h, \gamma}$, and saturations $s_{h, i}$ and $s_{h, \gamma}$, are piecewise constant and they are sought in the following spaces

$$
\begin{aligned}
& M_{h}^{i}=\left\{q_{h, i} \in L^{2}(\Omega) ; \forall K \in \mathcal{T}_{h}^{i},\left.q_{h, i}\right|_{K}=q_{K, i} \in \mathbb{R}\right\}, \\
& M_{h}^{\gamma}=\left\{q_{h, \gamma} \in L^{2}(\gamma) ; \forall \sigma \in \mathcal{T}_{h}^{\gamma},\left.q_{h, \gamma}\right|_{\sigma}=q_{\sigma, \gamma} \in \mathbb{R}\right\} .
\end{aligned}
$$

The degrees of freedom for the pressure and the saturation are their respective averages over the cells.

The approximate vector unknowns, total velocities $\mathbf{u}_{h, i}$ and $\mathbf{u}_{h, \gamma}$, and capillary diffusion velocities $\mathbf{r}_{h, i}$ and $\mathbf{r}_{h, \gamma}$, are piecewise polynomials sought in the following spaces

$$
\begin{aligned}
\mathbf{V}_{h}^{i} & =\left\{\mathbf{v}_{h, i} \in \mathbf{H}\left(\operatorname{div}, \Omega_{i}\right) ; \forall K \in \mathcal{T}_{h}^{i},\left.\mathbf{v}_{h, i}\right|_{K}=\mathbf{v}_{K, i} \in \mathbf{R T N}_{0}(K)\right\}, \\
\mathbf{V}_{h}^{\gamma} & =\left\{\mathbf{v}_{h, \gamma} \in \mathbf{H}(\operatorname{div}, \gamma) ; \forall \sigma \in \mathcal{T}_{h}^{\gamma},\left.\mathbf{v}_{h, \gamma}\right|_{\sigma}=\mathbf{v}_{\sigma, \gamma} \in \mathbf{R T}_{0}(\sigma)\right\},
\end{aligned}
$$

where $\mathbf{R T N}_{0}(K)$ and $\mathbf{R} \mathbf{T}_{0}(\sigma)$ are the local lowest-order Raviart-Thomas-Nédélec mixed finite element spaces [41, 44]. They are uniquely defined by their fluxes through faces in 3-D and through edges in 2-D.

\subsection{Time stepping}

The saturation equation and the pressure equation are solved sequentially. For the saturation equation we use an operator splitting in order to use different time steps for diffusion and for advection. For the diffusion step an implicit Euler method is used while the advection step is treated explicitly with a Godunov type method. 
Also the fracture and the surrounding matrix have very different physical properties, so one might like to use different time steps in the matrix domain and in the fracture, smaller time steps in the fractures of permeability higher than that in the matrix or on the contrary, possibly larger time steps in the fracture if it is a barrier. In this paper we have not pursued this idea, but have used the same time steps in the fracture as in the matrix. However, we refer the interested reader to the series of articles $[27,29,28]$, where a method for implementing this idea is described.

For simplicity of exposition we use a uniform time discretization of $\mathrm{N}$ intervals of the time interval of simulation $\left(0, T_{f}\right]$. Let $\Delta t=T_{f} / N$ and $t^{n}=n \Delta t$. It is straightforward to adapt the method to a nonuniform time discretization. The calculation is initialized by setting the values of $s_{h, i}^{0}$ to $s_{K, i}^{0}=\frac{1}{|K|} \int_{K} s_{0}$, for each $K \in \mathcal{T}_{h}^{i}, i=1,2$ and the values of $s_{h, \gamma}^{0}$ to $s_{\sigma, \gamma}^{0}=\frac{1}{|\sigma|} \int_{\sigma} s_{0}$, for each $\sigma \in \mathcal{T}_{h}^{\gamma}$.

Assuming we know at time $t^{n}$ the saturation $s_{h, i}^{n}$ in the subdomains $\Omega_{i}$ and $s_{h, \gamma}^{n}$ in the interface $\gamma$ we calculate the saturation $s_{h, i}^{n+1}, s_{h, \gamma}^{n+1}$ at time $t^{n+1}$ in two steps. In the first step we solve the pressure equation and in the second step we solve the saturation equation. Using operator splitting this second step is actually divided into a first substep during which we advance saturation through advection and a second step during which we advance saturation through diffusion.

\subsection{Step 1: The pressure equation}

Here we derive the approximation equations for the interface problem for the reduced fracture formulation of the pressure equation (55)-(57). Given saturations $s_{h i}^{n} \in M_{h}^{i}$ on the subdomains and $s_{h \gamma}^{n} \in M_{h}^{\gamma}$ on the interface from the previous time step $n \geq 1$ or from the initial data when $n=0$, we solve the pressure equation using a MFE method with non-overlapping domain decomposition [25, 37]. With the same notation for the Robin-to-Neumann operators for the approximation problem as for the continuous problem, the discrete form of the Robin-to-Neumann operator for the pressure equation is defined by

$$
\begin{aligned}
\mathcal{L}_{p i}^{R t N, n}: M_{h}^{\gamma} & \longrightarrow M_{h}^{\gamma}, \\
\lambda_{h, \gamma} & \longmapsto-\left.\left(\mathbf{u}_{h, i}^{n} \cdot \mathbf{n}_{i}\right)\right|_{\gamma},
\end{aligned}
$$


where $\lambda_{h, \gamma}$ is a pressure function given on the interface $\gamma$ and for $i=1,2$ the pair $\left(\mathbf{u}_{h, i}^{n}, p_{h, i}^{n}\right) \in$ $\mathbf{V}_{h}^{i} \times M_{h}^{i}$ is the solution of the subdomain problem

$$
\begin{aligned}
& \int_{\Omega_{i}} \nabla \cdot \mathbf{u}_{h, i}^{n} r=\int_{\Omega_{i}} q_{i} r, \quad \forall r \in M_{h}^{i}, \\
& \left.\int_{\Omega_{i}}\left(\widehat{\mathbf{K}}_{i}^{n}\right)^{-1} \mathbf{u}_{h, i}^{n}\right) \cdot \mathbf{v}-\int_{\Omega_{i}} p_{h, i}^{n} \nabla \cdot \mathbf{v}=\int_{\gamma} \bar{p}_{i, \gamma} \mathbf{v} \cdot \mathbf{n}_{i}+\int_{\Omega_{i}} \rho_{i}\left(s_{h, i}^{n}\right) \mathbf{u}_{G} \cdot \mathbf{v}, \quad \forall \mathbf{v} \in \mathbf{V}_{h}^{i}, \\
& \kappa\left(s_{h, \gamma}^{n}\right) \bar{p}_{i, \gamma}-\mathbf{u}_{h, i}^{n} \cdot \mathbf{n}_{i}=\kappa\left(s_{h, \gamma}^{n}\right)\left(\lambda_{h, \gamma}-\beta_{\gamma}\left(s_{h, \gamma}^{n}\right)+\beta_{i}\left(s_{h, i}^{n}\right)\right), \quad \text { on } \partial \Omega_{i} \cap \gamma,
\end{aligned}
$$

where $\left.\widehat{\mathbf{K}}_{i}^{n}=\mathbf{K}_{i} k_{i}\left(s_{h, i}^{n}\right)\right)$ and $\bar{p}_{i, \gamma} \in M_{h}^{\gamma}$ is an auxiliary unknown denoting a trace of the pressure on $\gamma \cap \partial \Omega_{i}$ while $\lambda_{h, \gamma}$ denotes an interface pressure in $\gamma$. Equation (64) enforces the transmission equation (38) and provides a Robin boundary condition on $\partial \Omega_{i} \cap \gamma$ for the subdomain problems.

The pressure equation formulated as an interface problem can be written as follows:

Find the pair $\left(\mathbf{u}_{h, \gamma}^{n}, p_{h, \gamma}^{n}\right) \in \mathbf{V}_{h}^{\gamma} \times M_{h}^{\gamma}$ such that

$$
\begin{aligned}
& \int_{\gamma}\left(\nabla_{\tau} \cdot \mathbf{u}_{h, \gamma}^{n}+\mathcal{L}_{p 1}^{R t N, n}\left(p_{h, \gamma}^{n}\right)+\mathcal{L}_{p 2}^{R t N, n}\left(p_{h, \gamma}^{n}\right)\right) r=\int_{\gamma} q_{h, \gamma}^{n} r, \quad \forall r \in M_{h}^{\gamma}, \\
& \int_{\gamma}\left(\left(\widehat{\mathbf{K}}_{\gamma}^{n}\right)^{-1} \mathbf{u}_{h, \gamma}^{n}\right) \cdot \mathbf{v}-\int_{\gamma} p_{h, \gamma}^{n} \nabla \cdot \mathbf{v}=\int_{\gamma} \rho_{\gamma}\left(s_{h, \gamma}^{n}\right) \mathbf{u}_{G, \tau} \cdot \mathbf{v}, \quad \forall \mathbf{v} \in \mathbf{V}_{h}^{\gamma},
\end{aligned}
$$

where $\widehat{\mathbf{K}}_{\gamma}^{n}=\mathbf{K}_{\gamma} k_{\gamma}\left(s_{h, \gamma}^{n}\right)$.

The Dirichlet-to-Neumann operators $\mathcal{L}_{p i}^{R t N, n}$ are affine and can be split into their linear parts and their constant parts. In this way we obtain a linear problem which can be solved using an iterative method where each iteration involves solving the subdomain problems (62), (63), (64) on $\Omega_{1}$ and $\Omega_{2}$.

\subsection{Step 2: The saturation equation}

The calculation of the saturation is split into two substeps, an advection substep followed by a diffusion substep.

\subsubsection{Advection substep}

The time step $\Delta t=t^{n+1}-t^{n}, n=1, \cdots, N-1$, is divided into $L$ advection time steps of length $\Delta t_{a}=t^{n, l+1}-t^{n, l}, l=0, \cdots, L-1, n=1, \cdots, N-1$, where $t^{n, l}=t^{n}+l \Delta t_{a}, l=0, \cdots, L-1 ; n=$ $1, \cdots, N-1$. For simplicity we have taken $L$ independent of the subdomain and of whether we are in the fracture or in the matrix though one could of course choose a different number $L$ for each subdomain and for the fracture. 
For values of the saturations $s_{h, i}^{n}, i=1,2$ and $s_{h, \gamma}^{n}$, either given as initial values (if $n=0$ ) or obtained from the the $n^{\text {th }}$ diffusion step (if $n \geq 1$ ), the advection step is initialized by setting $s_{h, i}^{n, 0}=s_{h, i}^{n}$ and $s_{h, \gamma}^{n, 0}=s_{h, \gamma}^{n}$, and, for $l=1, \cdots, L$, intermediate saturations $s_{h, i}^{n, l}, i=1,2$ and $s_{h, \gamma}^{n, l}$ are calculated using a first order cell-centered finite volume method of Godunov type.

In the subdomains. In the subdomains $\Omega_{i}, i=1,2$ we write

$$
\int_{K} \Phi_{K} \frac{s_{K, i}^{n, l+1}-s_{K, i}^{n, l}}{\Delta t_{a}^{i}}+\sum_{\sigma \in \mathcal{F}_{K}}|\sigma| \varphi_{\sigma}^{n, l}=0, \quad \forall K \in \mathcal{T}_{h}^{i}, \quad l=1, \cdots, L-1,
$$

where $|\sigma| \varphi_{\sigma}^{n, l}$ is an approximation of the advection flux $\int_{\sigma} f_{a}^{n}$, with

$$
f_{a}^{n}(s)=\mathbf{f}^{n}(s) \cdot \mathbf{n}_{\sigma}=f_{w}(s)\left(\mathbf{u}^{n}+f_{G w}(s) \mathbf{u}_{G}\right) \cdot \mathbf{n}_{\sigma} .
$$

$\varphi_{\sigma}^{n, l}$ is a function of the two values of the saturation on the two sides of $\sigma$ with $n_{\sigma}$ the normal to $\sigma$ chosen as pointing from one side of $\sigma$ denoted by $\sigma^{-}$to the other side $\sigma^{+}$. If $\sigma \subset \Omega_{i} n_{\sigma}$ points towards the outside of $\Omega_{i}$. Then

$$
\varphi_{\sigma}^{n, l}=\varphi_{\mu \nu}^{n}\left(s_{\sigma^{-}}^{n, l}, s_{\sigma^{+}}^{n, l}\right)
$$

where $s_{\sigma^{-}}^{n, l}$ and $s_{\sigma^{+}}^{n, l}$ are the values of the saturation on the two sides of $\sigma$ and the indices $\mu$ and $v$ refer to the rock types in $\sigma^{-}$and in $\sigma^{+}, \mu, v \in\{1,2, \gamma\}$. Also $\mu=v$ if there is no change of rock type. The function $\varphi_{\mu \nu}^{n}$ depends on the flux functions associated with the rock types on the two sides of $\sigma$ which we denote

$$
f_{a j}^{n}(s)=\mathbf{f}_{j}^{n}(s) \cdot \mathbf{n}_{\sigma}=f_{w j}(s)\left(\mathbf{u}_{h}^{n}+f_{G w j}(s) \mathbf{u}_{G}\right) \cdot \mathbf{n}_{\sigma}, \quad j=1,2, \gamma .
$$

We give here the two most frequently used definitions for $\varphi_{\mu v}$. The first one is an extension of the Godunov flux to the case with a change of rock type given in [1]:

$$
\begin{aligned}
& \text { If } f_{a \mu} \text { and } f_{a v} \text { have one minimum: } \quad \varphi_{\mu \nu}(a, b)=\max \left\{f_{a \mu}\left(\max \left\{a, \xi_{f_{\mu}}\right\}\right), f_{a v}\left(\min \left\{\xi_{f_{v}}, b\right\}\right)\right\} \\
& \text { with } \xi_{f_{j}}=\operatorname{argmin} f_{a j}, j=\mu, v \text {, } \\
& \text { If } f_{a \mu} \text { and } f_{a v} \text { have one maximum: } \quad \varphi_{\mu \nu}(a, b)=\min \left\{f_{a \mu}\left(\min \left\{a, \xi_{f_{\mu}}\right\}\right), f_{a \gamma}\left(\max \left\{\xi_{f_{v}}, b\right\}\right)\right\} \\
& \text { with } \xi_{f_{j}}=\operatorname{argmax} f_{a j}, j=\mu, v \text {. }
\end{aligned}
$$

When there is no change of rock type these formulas are equivalent to the standard Godunov flux. 
A second definition for $\varphi_{\mu \nu}$ is the phase upstream numerical flux [9]:

$$
\varphi_{\mu v}(a, b)=\varphi_{w, \sigma}=f_{w}^{\star}\left(\mathbf{u}_{h}^{n}+f_{G w}^{\star} \mathbf{K} \mathbf{u}_{G}\right) \cdot \mathbf{n}_{\sigma},
$$

where $f_{w}^{\star}, f_{G w}^{\star}$ are calculated with formulas (7) and (13) using the mobility $k_{w}$ (resp. $k_{n w}$ ) calculated with the upstream mobility and saturation values with respect to the flow of the wetting (resp. non-wetting) phase:

$$
k_{\ell}^{\star}=\left\{\begin{array}{ll}
k_{\ell, \mu}(a) & \text { if }\left(\mathbf{u}_{h}+f_{G w}^{\star} \mathbf{K} \mathbf{u}_{G}\right) \cdot \mathbf{n}_{\sigma}>0, \\
k_{\ell, v}(b) & \text { if }\left(\mathbf{u}_{h}+f_{G n w}^{\star} \mathbf{K} \mathbf{u}_{G}\right) \cdot \mathbf{n}_{\sigma} \leq 0,
\end{array} \quad \ell=w, n w .\right.
$$

The phase upstream flux is convergent when there is no change of rock type [15] but it can fail for some configurations of relative mobility curves [38]. The extended Godunov flux (68) solves the problem correctly for more cases, but still, in pathological cases, it can also fail and in [7] it is shown how to proceed correctly. This solution requires also the use of capillarity curves.

In the fracture. Equations in $\gamma$ are similar to that in the subdomains except they are now in two dimensions instead of three. Also there is now a right-hand side representing the contributions of the subdomains to the flow in the fracture:

$$
\int_{\sigma} \Phi_{\sigma} \frac{s_{\gamma \sigma}^{n, l+1}-s_{\gamma \sigma}^{n, l}}{\Delta t_{a}^{i}}+\sum_{e \in \mathcal{E}_{\sigma}}|e| \varphi_{e}^{n, l}=\sum_{i=1,2}|\sigma| \varphi_{\sigma, i}^{n, l}, \quad \forall \sigma \in \mathcal{T}_{h}^{\gamma}, \quad l=1,, \cdots, L-1 .
$$

$|e| \varphi_{e}^{n, l}$ is an approximation of the advection flux through the edge $e, \int_{e} f_{a \gamma}^{n}$, with

$$
f_{a \gamma}^{n}(s)=\mathbf{f}_{\gamma}(s)^{n} \cdot \mathbf{n}_{e}=f_{w \gamma}(s)\left(\mathbf{u}_{h i}^{n}+f_{G w \gamma}(s) \mathbf{u}_{G, \tau}\right) \cdot \mathbf{n}_{e} .
$$

As in the subdomains $\varphi_{e}^{n, l}$ is a function of the two values of the saturation adjacent to $e=\sigma^{-} \mid \sigma^{+}$:

$$
\varphi_{e}^{n, l}=\varphi_{\gamma}^{n}\left(s_{\gamma \sigma^{-}}^{n, l}, s_{\gamma \sigma^{+}}^{n, l}\right),
$$

where $s_{\gamma \sigma^{-}}^{n, l}$ and $s_{\gamma \sigma^{+}}^{n, l}$ are the upstream and downstream cell-values of the saturation with respect to the direction pointed by $\mathbf{n}_{e}$. The function $\varphi_{\gamma}^{n}$ is defined by the same Godunov or phase by phase upstream formula except that the function $f_{a i}^{n}$ is replaced by $f_{a \gamma}^{n}$. If there is a change of rock type inside the fracture it can be dealt in the same way as at the interface between the fracture and the subdomains. 


\subsubsection{Diffusion substep}

Given the saturations on the subdomains $s_{h i}^{n, L} \in M_{i}^{h}, i=1,2$ and on the interface $\theta_{h, \gamma}^{n, L}$ given from the advection step we calculate the saturations $s_{h i}^{n+1} \in M_{i}^{h}, i=1,2$, and $\theta_{h, \gamma}^{n+1}$ by solving the diffusion equations formulated as an interface problem using domain decomposition as we did for the pressure equations, except that now the Robin to Neumann operator as well as the interface problem is nonlinear.

For the diffusion equation we consider the discrete form of the Robin to Neumann operator defined by

$$
\begin{aligned}
\mathcal{L}_{s i}^{R t N, n}: M_{h}^{\gamma} & \longrightarrow M_{h}^{\gamma}, \\
\theta_{h, \gamma} & \longmapsto-\left.\left(\mathbf{r}_{h, i}^{n} \cdot \mathbf{n}_{i}\right)\right|_{\gamma},
\end{aligned}
$$

where $\theta_{h, \gamma}$ is a saturation function given on the interface $\gamma$ and the pair $\left(\mathbf{r}_{h, i}^{n+1}, s_{h, i}^{n+1}\right) \in \mathbf{V}_{h}^{i} \times M_{h}^{i}$ is, for $i=1,2$, the solution of the subdomain problem

$$
\begin{aligned}
& \int_{\Omega_{i}} \Phi_{i} \frac{s_{h, i}^{n+1}-s_{h, i}^{n, L}}{\Delta t} r+\int_{\Omega_{i}} \nabla \cdot \mathbf{r}_{h, i}^{n} r=\int_{\Omega_{i}} q_{w i} r \quad \forall r \in M_{h}^{i}, \\
& \int_{\Omega_{i}}\left(\mathbf{K}_{i}^{-1} \mathbf{r}_{h, i}^{n}\right) \cdot \mathbf{v}-\int_{\Omega_{i}} \alpha_{i}\left(s_{h, i}^{n}\right) \nabla \cdot \mathbf{v}=\int_{\gamma} \alpha_{i}\left(\bar{s}_{i, \gamma}^{n+1}\right) \mathbf{v} \cdot \mathbf{n}_{i} \quad \forall \mathbf{v} \in \mathbf{V}_{h}^{i}, \\
& \mathbf{r}_{h, i}^{n+1} \cdot \mathbf{n}_{i}-\kappa_{w}\left(\theta_{h, \gamma}\right) \frac{\pi_{i}\left(\bar{s}_{i, \gamma}^{n+1}\right)}{2}=-\kappa_{w}\left(\theta_{h, \gamma}\right) \frac{\pi_{\gamma}\left(\theta_{h, \gamma}\right)}{2}, \quad \text { on } \partial \Omega_{i} \cap \gamma,
\end{aligned}
$$

where $\bar{s}_{i, \gamma}^{n+1} \in M_{h}^{\gamma}$ is an auxiliary unknown denoting a trace of the saturation on $\partial \Omega_{i} \cap \gamma$ while $\theta_{h, \gamma}$ denotes an interface saturation in $\gamma$.

Equation (72) provides a nonlinear Robin boundary condition on $\partial \Omega_{i} \cap \gamma$ to the subdomain problem. It enforces the transmission equation (45). Indeed since $\mathbf{u}_{w i} \cdot \mathbf{n}_{i}=\left(\mathbf{r}_{i}+\mathbf{f}_{i}\right) \cdot \mathbf{n}_{i}$ we notice that in Equation (45) there are two advective terms $\mathbf{f}_{i} \cdot \mathbf{n}_{i}$ on the right hand side and $f_{w \gamma}\left(s_{\gamma}\right) \mathbf{u}_{i} \cdot \mathbf{n}_{i}$ on the left hand side which both represent the advective flux through $\partial \Omega_{i} \cup \gamma$. Therefore they cancelled out during the advective step in order to preserve mass conservation and we obtain the transmission equation (72) for the diffusion step.

Then the saturation equations for the diffusion step formulated as an interface problem can be written as to calculate the pair $\left(r_{h, \gamma}^{n+1}, s_{h, \gamma}^{n+1}\right) \in \mathbf{V}_{h}^{\gamma} \times M_{h}^{\gamma}$ satisfying

$$
\begin{aligned}
& \int_{\gamma} \Phi_{\gamma} \frac{s_{h, \gamma}^{n+1}-s_{h, \gamma}^{n, L}}{\Delta t} r+\int_{\gamma} \nabla \cdot \mathbf{r}_{h, \gamma}^{n+1} r+\int_{\gamma} \sum_{i=1,2} \mathcal{L}_{s i}^{R t N, n}\left(s_{h, \gamma}^{n+1}\right) r=\int_{\gamma} q_{w \gamma} r \quad \forall r \in M_{\gamma}^{i}, \\
& \int_{\gamma} \mathbf{K}_{\gamma}^{-1} \mathbf{r}_{h, \gamma}^{n+1}, \mathbf{v}-\int_{\gamma} \alpha_{\gamma}\left(s_{h, \gamma}^{n+1}\right) \nabla \cdot \mathbf{v}=0 \quad \forall \mathbf{v} \in \mathbf{V}_{\gamma}^{i} .
\end{aligned}
$$




\section{Implementation}

Using the close relationship between mixed finite elements and cell-centered finite volumes on triangles $[2,48,47,19]$ the interface problems on $\gamma$ for the pressure equation (65), (66) and for the diffusion part of the saturation equation (73),(66) were actually implemented as a triangular cell-centered finite volume method while the Robin to Neumann operators $\mathcal{L}_{p i 1}^{R t N, n}$ and $\mathcal{L}_{s i}^{R t N, n}, i=1,2$ were implemented using tetrahedral mixed finite elements of lowest order.

Then the interface reduced pressure problem (66), (65) can be rewritten in an operator primal formulation as

$$
\nabla_{\tau} \cdot\left(-\mathbf{K}_{\gamma} k_{\gamma}\left(s_{h, \gamma}^{n}\right) \nabla_{\tau} p_{h, \gamma}^{n}\right)+\left(\mathcal{L}_{p 1}^{R t N, n}+\mathcal{L}_{p 2}^{R t N, n}\right)\left(p_{h, \gamma}^{n}\right)=q_{h, \gamma}^{n}
$$

It was shown in [6] that a good preconditioner to solve this linear problem by a conjugate gradient method is the inverse of the operator $\nabla_{\tau} \cdot\left(-\mathbf{K}_{\gamma} k_{\gamma}\left(s_{h, \gamma}^{n}\right) \nabla_{\tau} \cdot\right)$.

Similarly, in the calculation of the saturation, the interface reduced diffusion problem (73), (74) can be rewritten in an operator primal formulation as

$$
\Phi_{\gamma} \frac{s_{h, \gamma}^{n+1}-s_{h, \gamma}^{n, L}}{\Delta t}+\nabla_{\tau} \cdot\left(-\mathbf{K}_{\gamma} \nabla \alpha_{\gamma}\left(s_{h, \gamma}^{n+1}\right)\right)+\left(\mathcal{L}_{s 1}^{R t N, n}+\mathcal{L}_{s 2}^{R t N, n}\right)\left(s_{h, \gamma}^{n+1}\right)=q_{w h, \gamma}^{n} .
$$

At each time step $n+1$, this nonlinear interface system is solved using an inexact Newton GMRES approach. At each Newton iteration the linear system is solved inexactly using GMRES and the jacobian matrix-vector product is approximated by forward finite differences (Jacobian Free Newton Krylov (JFNK) method, see [34]).

Our sequential method is summarized by the following steps:

$\overline{\text { Algorithm. }} \overline{n=0: s_{h, i}^{0}, s_{h, \gamma}^{0} \text { are given. }}$
For $\mathrm{n}=1, \ldots, \mathrm{N}$ do

1. Solve the interface pressure equation (65),(66) to obtain $p_{h, \gamma}^{n}$ and $p_{h, i}^{n}$,

2. Perform $L$ explicit advection steps of the saturation equation in the subdomains and in the interface $\gamma$ as described in section 7.4.1 to obtain $s_{h, i}^{n, L}$ and $s_{h, \gamma}^{n . L}$.

3. Solve the interface diffusion problem (73),(74) to obtain $s_{h, i}^{n+1}$ and $s_{h, \gamma}^{n+1}$.

End do 
Our code is written in Matlab using programs from the library MRST [36]. Concerning meshes, the interface $\gamma$ and the boundary $\partial \Omega$ were discretized with the three-dimensional surface meshing code BLSURF [13]. From this surface meshes three-dimensional volumetric meshes were produced by the code GHS3D [22].

\section{Numerical experiments}

We consider displacements of a nonwetting fluid by a wetting fluid in a porous medium which is a cubic domain $\Omega$ of dimensions $20 \times 20 \times 20$ [m]. The wetting fluid is injected through one vertical face of this cube and the fluids exit the cube through the opposite face. On the four other faces a no flow condition is imposed. See Fig. 5.

We will consider several configurations for the fractures, first with a single fracture and latter with intersecting fractures. These fractures may have a larger permeability than the matrix rock or may be barriers. It is important to notice that in our experiments, even though the interface $\gamma$ goes all the way to the boundary $\partial \Omega$, the fracture itself does not in the horizontal direction and stops $1 / 10$ of a side length from each of the vertical boundary faces. In the part of $\gamma$ which is not the actual fracture the rock type is that of the rock matrix. Consequently we encounter two changes of rock type when moving horizontally along $\gamma$. (Alternatively one could impose a noflow condition on the bounday of the actual fracture. However with our domain decomposition approach it is convenient to simply extend the fracture up to the domain boundary.)

In all the experiments presented here the absolute permeability tensors $\mathbf{K}$ are actually scalar absolute permeabilities $K$, and the Muelam-Van Genuchten model is used for the relative permeability and capillary pressure curves. Relative permeabilities as functions of the wetting phase saturation are, for both the rock matrix and the fracture,

$$
k_{w}\left(s_{w}\right)=\sqrt{s_{w}}\left[1-\left(1-s_{w}^{n}\right)^{m}\right]^{2}, \quad k_{n w}\left(s_{w}\right)=\left(1-s_{w}\right)^{2}\left(1-s_{w}^{n}\right)^{2 m}
$$

with $n=2.8, m=1-1 / n$. The capillary pressure function is of the form

$$
\pi(s)=\sqrt{\frac{\Phi}{K}}\left((1-s)^{-1 / m}-1\right)^{1 / n} .
$$

All of our tests include gravity effects. 


\subsection{Experiment with a single fracture}

First, we consider a simple numerical experiment with a single vertical fracture located in the middle of the domain, perpendicular to the injection and production faces of $\Omega$. See Fig. 5 left. The width $d$ of the fracture is two thousand times smaller than the length of the edges of $\Omega$. Fig. 5 right shows the capillary pressure curves.
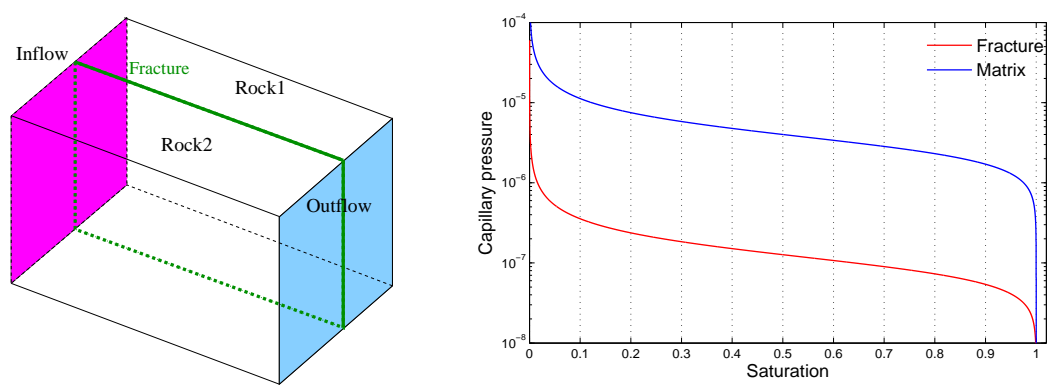

Figure 5: Left: Single fracture experiments: No flow except through the magenta inflow region and the blue outflow region. Right: Capillary pressure curves.

The boundary conditions for the (global) pressure on the inflow and outflow boundaries are 50 and 0 psi respectively. The saturation boundary conditions are $s_{w}=1$ on the inflow boundary. On the outflow boundary the saturation at time $t^{n+1}$ is set to be equal to that inside the closest cell at time $t^{n}$. This condition is a simple device for approximating a transparent boundary condition. The rock parameters we use are the same in the two subdomains. The fluid and medium properties are provided in Table 1.

Table 1: Data for example 1

\begin{tabular}{|l|}
\hline Rock and fluid propreties \\
\hline Matrix properties: $\Phi_{m}=0.1, K_{m}=1 \mathrm{md}$ \\
Fracture properties: $\Phi_{f}=0.7, K_{f}=10^{3} \mathrm{md}$ \\
Fluid properties: $\mu_{w}=1 \mathrm{cp}, \rho_{w}=1000 \mathrm{~kg} / \mathrm{m}^{3}$ \\
$\mu_{n w}=0.45 \mathrm{cp}, \rho_{n w}=660 \mathrm{~kg} / \mathrm{m}^{3}$ \\
\hline
\end{tabular}

Fig. 6 shows the saturation of the wetting phase at two different times. At left the saturation $s_{w}$ is shown on three faces of the boundary of $\Omega$ and at right we show $s_{w}$ on the bottom face of $\Omega$. 
Note that the saturation in the fracture itself cannot be seen in the pictures. For this calculation the 2-D mesh for $\gamma$ together with the boundary of $\Omega$ has 880 triangles and from this 2-D mesh was produced a volumetric mesh with 72000 tetrahedra.
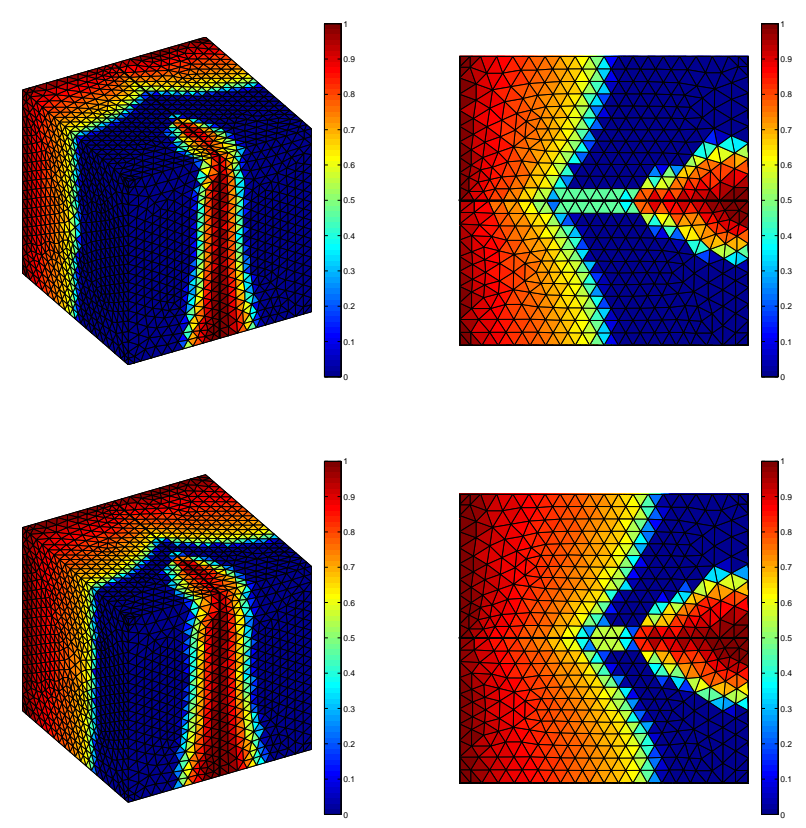

Figure 6: A single vertical fracture: saturation of the injected wetting fluid at two different times. At left saturation on $\partial \Omega$. At right saturation on the bottom face of $\Omega$.

From equations (37), (41), (44) and (49) we see that gravity effects are proportional to the absolute permeability and therefore are more important in the fracture than in the rock matrix. This explains why we see in Fig. 6 an accumulation of the wetting fluid at the bottom of $\Omega$ near the fracture.

We observe also a cusp in the saturation isolines near the entrance of the fracture as the wetting phase is drawn into the more permeable fracture. In the vicinity of the fracture exit however, the wetting phase fluid accumulates as it enters the less permeable rock matrix.

Fig. 7 shows the calculated saturation of the injected wetting phase along a line orthogonal to the fracture with a comparison between the reduced model and a three-dimensional model where the fracture width is discretized with six cells. In both models there are two saturation 


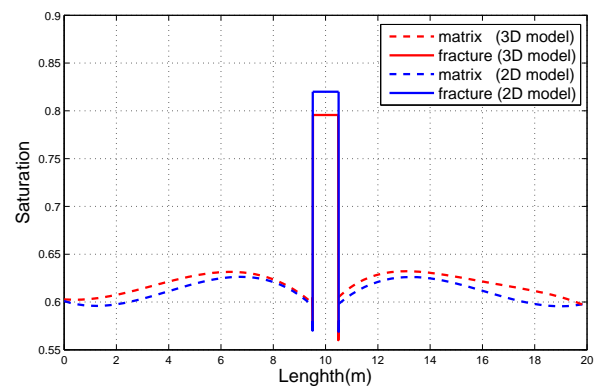

Figure 7: Saturation of the injected wetting fluid along a line orthogonal to the fracture with a comparison between the reduced fracture model (2D model) and a full three-dimensional model (3D model)

values at the fracture-matrix interface to take into account the saturation discontinuity due to the change of capillary pressure curve. In Fig. 7 the width of the fracture is blown up and the shown saturation values inside the fracture is $s_{h, \gamma}$ for the reduced model and the average of the six saturation cell values for the three-dimensional model. We note that the two models give similar results. We observe inverted spikes in the saturation curves at the interfaces between the fracture and the matrix. These are physical for the following reason. Since the permeability in the fracture is higher than in the matrix, due to equation (75) the capillarity pressure curve for the fracture is above that for the matrix (see Fig. 5 right). Therefore at the fracture-matrix interface, continuity of the capillary pressure implies a smaller saturation in the fracture than in the matrix. On the other hand, since the displacement of the non wetting phase is faster in the fracture than in the matrix the overall saturation in the fracture is larger than in the matrix, which induces the inverted spikes observed in Fig. 7.
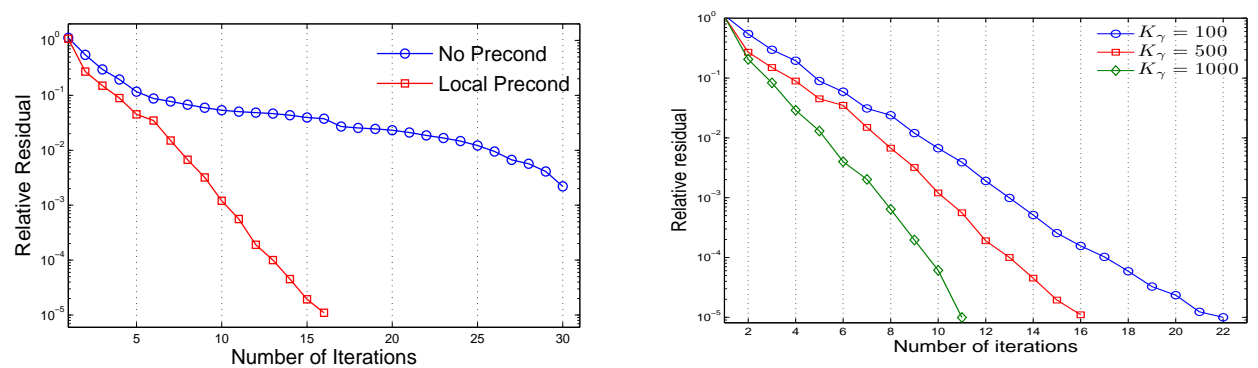

Figure 8: Efficiency of the CG preconditioner. 
For this same example, we show the effects of the preconditioners presented briefly in Section 8. For the interface reduced pressure equation (65), (66) which is linear the preconditioner for the conjugate gradient method is the inverse of the fracture operator and Fig. 8 shows the resulting improvement in the convergence curves. The improvement increases with the value of the absolute permeability in the fracture.

For the interface reduced nonlinear saturation diffusion equation (73), (74) the system is solved using inexact Newton GMRES with the JFNK method. Fig. 9 shows the effect of the method on GMRES and on Newton iterations.
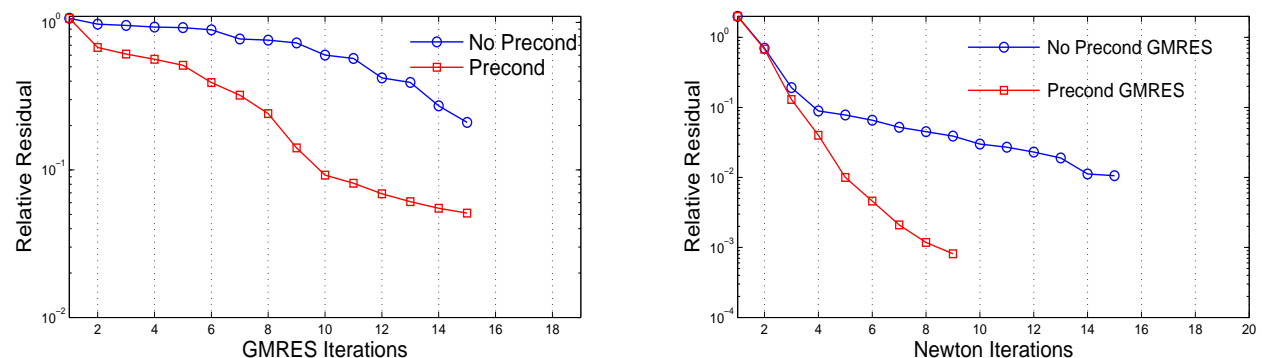

Figure 9: Effect of the preconditioner on GMRES and Newton convergences. 

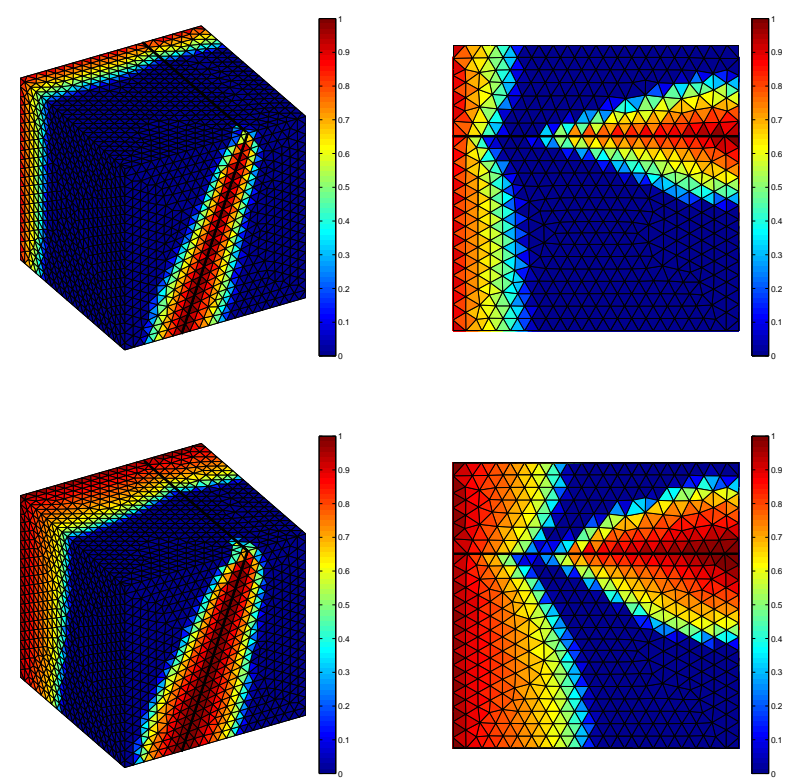

Figure 10: Inclined fracture: saturation of the injected wetting fluid at two different times. At left saturation on $\partial \Omega$. At right saturation on the bottom face of $\Omega$.

Figure 10 shows the results of an experiment identical to the previous one, that of Fig. 6 , except for the fact that in this experiment the fracture is inclined. Gravity no longer acts in a direction parallel to the fracture. The results differ but are qualitatively similar to that for the previous example.

\subsection{An experiment with three intersecting high permeability fractures}

We consider now a cubic fractured medium which is divided into three subdomains by three fractures connected along a straight line.

First we consider the case where the parameters in the subdomains and in fractures 1 and 3 are the same as for the previous experiments, but fracture 2 is five times more permeable than the two other fractures. See Fig. 11 on the left for the geometry, and on the right for the numerical results. One can observe that the flow is faster in fracture 2 so that it accumulates more at its endline when it runs into higher permeability. 

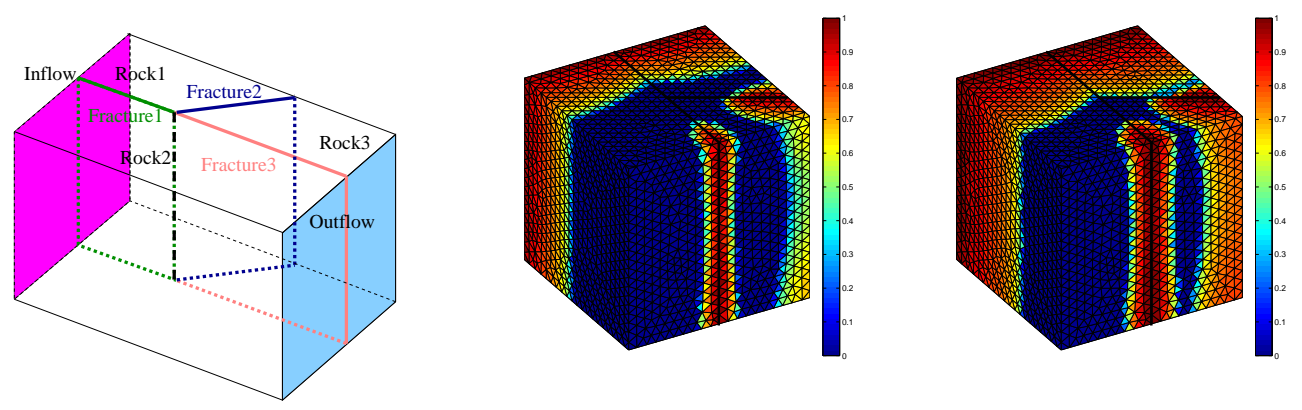

Figure 11: Left: Three intersecting fractures, no flow except through the magenta inflow region and the blue outflow region, higher permeability in fracture 2 than in fractures 1 and 3. Right: saturation at two different times.

\subsection{An experiment with three intersecting fractures, one a barrier}

In a last example, we introduce one barrier. The geometry is shown in Fig. 12 left. Unlike for the fractures in the previous examples, the part of the boundary of the barrier that is near the boundary of the domain lies actually on the boundary of the domain. The barrier has a low permeability $\left(10^{-5} \mathrm{md}\right)$ compared to the other fractures $\left(10^{3} \mathrm{md}\right)$. We assume that there is no flow into the barrier from its edge in common with the other two fractures. The volumetric mesh has 72137 tetrahedra and the two-dimensional mesh for $\gamma$ has 880 triangles. The evolution of the saturation of the injected fluid is presented in Fig. 12 right. We observe that the injected fluid does not cross the barrier in the left part of the domain, and that it is captured in the matrix subdomains and in fractures 1 and 2 .
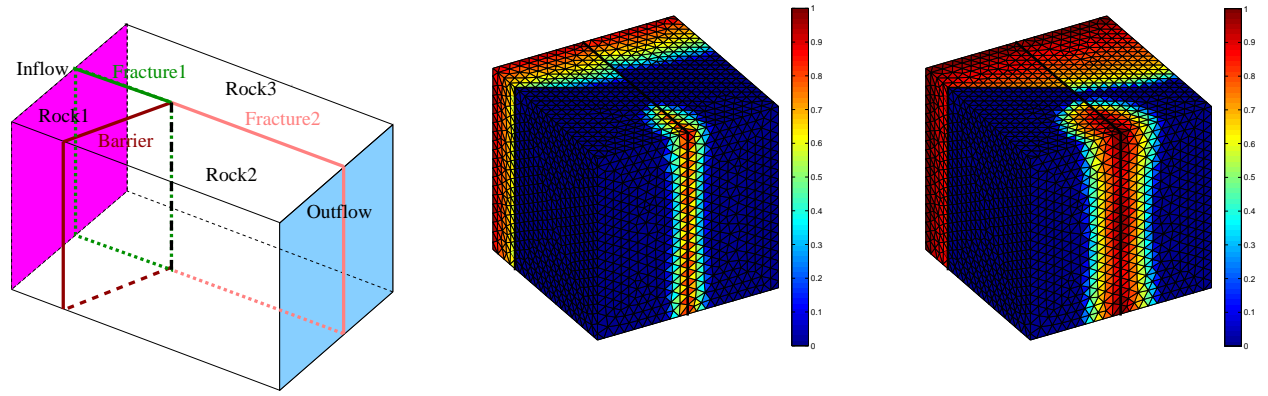

Figure 12: Left: Two fractures and a barrier, no flow except through the magenta inflow region and the blue outflow region. Right: saturation at two different times. 


\section{Conclusion}

In this paper we presented a model for two-phase flow in porous media with fractures. The model is a reduced fracture model, i.e. a discrete fracture model in which the fractures are treated as interfaces but which allow fluid flow exchange between the fractures and the rock matrix. This model takes into account changes in the relative permeability and capillary pressure curves and can handle barriers as well as fractures with large permeabilities. The model is discretized with mixed finite elements and cell-centered finite volume methods. Some numerical experiments were shown in order to check the validity of the method. These are preliminary experiments. One would like to do more experiments with more complex fracture configurations and to study problems with capillary barriers. An important improvement would be to be able to use different time steps in the fractures and in the matrix rock. Introducing meshes which do not match up at the interface between the fracture and the matrix would be very useful in order to minimize meshing difficulties in complex geometry. The construction of a posteriori error estimates would help for optimizing space and time discretizations. Then one could contemplate tackling more complex flow such as compressible two-phase flow and two-phase, two-component flow and transport problems.

[1] Adimurthi, J. Jaffré, G.V. Gowda, Godunov-type methods for conservation laws with a flux function discontinuous in space, SIAM J. Numer. Anal. 42 (2004) 179-208.

[2] P. Agouzal, J. Baranger, J.F. Maître, F. Oudin, Connection between finite volumes and mixed finite element methods for a diffusion problem with nonconstant coefficients, with application to convection-diffusion, East-West J. Numer. Anal. 3 (1995) 237-254.

[3] C. Alboin, J. Jaffré, J.E. Roberts, Domain decomposition for flow in porous media with fractures, in: C.H. Lai, P.E. Bjorstad, M. Cross (Eds.), Domain Decomposition Methods in Sciences and Engineering, Domain Decomposition Press, 1999, pp. 371-379.

[4] C. Alboin, J. Jaffré, J.E. Roberts, C. Serres, Modeling fractures as interfaces for flow and transport in porous media, in: Z. Chen, R. Ewing (Eds.), Fluid Flow and Transport in Porous Media : Mathematical and Numerical Treatment, volume 295 of Contemporary Mathematics, American Mathematical Society, 2002, pp. 13-24.

[5] C. Alboin, J. Jaffré, J.E. Roberts, X. Wang, C. Serres, Domain decomposition for some transmission problems in flow in porous media, in: Z. Chen, R. Ewing, Z.C. Shi (Eds.), Numerical Treatment of Multiphase Flows in Porous Media, Springer, 2000, pp. 22-34.

[6] L. Amir, M. Kern, V. Martin, J.E. Roberts, Décomposition de domaine pour un milieu poreux fracturé: un modèle en $3 d$ avec fractures qui s' intersectent, Arima 5 (2006) 11-25.

[7] B. Andreianov, C. Cancès, A phase-by-phase upstream scheme that converges to the vanishing capillarity solution for countercurrent two-phase flow in two-rock media, Computat. Geosci. 18 (2014) 211-226. 
[8] P. Angot, F. Boyer, F. Hubert, Asymptotic and numerical modelling of flows in fractured porous media, ESAIM: Math. Model. Numer. Anal. 43 (2009) 239-275.

[9] K. Aziz, A. Settari, Petroleum Reservoir Simulation, volume 476, Applied Science Publishers London, 1979.

[10] R. Baca, R. Arnett, D.W. Langford, Modelling fluid flow in fractured-porous rock masses by finite element techniques, Int. J. Numer. Meth. Fluids 4 (1984) 337-348.

[11] P. Bastian, Z. Chen, R. Ewing, R. Helmig, H. Jakobs, V. Reichenberger, Numerical simulation of multiphase flow in fractured porous media, in: Numerical Treatment of Multiphase Flows in Porous Media, Springer, 2000, pp. $50-68$.

[12] I. Bogdanov, V. Mourzenko, J. Thovert, P. Adler, Two-phase flow through fractured porous media, Phystcal Review E 68 (2003) 1-24.

[13] H. Borouchaki, P. Laug, P.L. George, Parametric surface meshing using a combined advancing-front - generalizedDelaunay approach, Int. J. Numer. Meth. Eng. 49 (2000) 233-259.

[14] A. Bourgeat, A. Hidani, A result of existence for a model of two-phase flow in a porous medium made of different rock types, Appl. Anal. 56 (1995) 381-399.

[15] Y. Brenier, J. Jaffré, Upstream differencing for multiphase flow in reservoir simulation, SIAM J. Numer. Anal. 28 (1991) 685-696.

[16] K. Brenner, M. Groza, C. Guichard, R. Masson, Vertex approximate gradient scheme for hybrid dimensional twophase Darcy flows in fractured porous media, ESAIM: Math. Model. Numer. Anal. 49 (2015) 303-330.

[17] G. Chavent, A new formulation of diphasic incompressible flows in porous media, in: Applications of Methods of Functional Analysis to Problems in Mechanics, Springer, 1976, pp. 258-270.

[18] G. Chavent, J. Jaffré, Mathematical Models and Finite Elements for Reservoir Simulation, North Holland, 1986.

[19] J. Droniou, R. Eymard, T. Gallouët, R. Herbin, A unified approach to mimetic finite difference, hybrid finite volume and mixed finite volume methods, Math. Mod. Meth. Appl. Sci. 20 (2010) 265-295.

[20] I. Faille, A. Fumagalli, J. Jaffré, J.E. Roberts, Model reduction and discretization using hybrid finite volumes for flow in porous media containing faults, Computational Geosciences (2016) 1-23. URL: http://dx.doi.org/10.1007/s10596-016-9558-3. doi:10.1007/s10596-016-9558-3.

[21] L. Formaggia, A. Fumagalli, A. Scotti, P. Ruffo, A reduced model for Darcy's problem in networks of fractures, ESAIM: Math. Model. Numer. Anal. 48 (2014) 1089-1116.

[22] P. Frey, P.L. George, Mesh Generation, ISTE, Wiley, 2008.

[23] N. Frih, V. Martin, J.E. Roberts, A. Saâda, Modeling fractures as interfaces with nonmatching grids, Computat. Geosci. 16 (2012) 1043-1060.

[24] A. Fumagalli, A. Scotti, A numerical method for two-phase flow in fractured porous media with non-matching grids, Adv. Water Resour. 62 (2013) 454-464.

[25] R. Glowinski, M.F. Wheeler, Domain decomposition and mixed finite element methods for elliptic problems, in: R. Glowinski, G. Golub, T.F. Chan, J. Periaux (Eds.), First International Symposium on Domain Decomposition Methods for Partial Differential Equations, SIAM, 1988, pp. 144-172.

[26] H. Haegland, A. Assteerrawatt, H.K. Dahle, G.T. Eigestad, R. Helmig, Comparison of cell- and vertex-centered discretization methods for flow in a two-dimensional discrete-fracture-matrix system, Adv. Water Resour. 32 (2009) 1740-1755. 
[27] T.T.P. Hoang, J. Jaffré, C. Japhet, M. Kern, J.E. Roberts, Space-time domain decomposition methods for diffusion problems in mixed formulations, SIAM J. Numer. Anal. 51 (2013) 3532-3559.

[28] T.T.P. Hoang, C. Japhet, M. Kern, J.E. Roberts, Space-time domain decomposition methods for advection-diffusion problems in mixed formulations, Math. Comput. Simulat. This issue (2016).

[29] T.T.P. Hoang, C. Japhet, M. Kern, J.E. Roberts, Space-time domain decomposition methods for reduced fracture models in mixed formulation, SIAM J. Numer. anal. 54 (2016) 288-316.

[30] H. Hoteit, A. Firoozabadi, An efficient numerical model for incompressible two-phase flow in fractured media, Adv. Water Resour. 31 (2008) 891-905.

[31] H. Hoteit, A. Firoozabadi, Numerical modeling of two-phase flow in heterogeneous permeable media with different capillarity pressures, Adv. Water Resour. 31 (2008) 56-73.

[32] J. Jaffré, M. Mnejja, J.E. Roberts, A discrete fracture model for two-phase flow with matrix-fracture interaction, Procedia Computer Science 4 (2011) 967-973.

[33] M. Karimi-Fard, A. Firoozabadi, A. Numerical simulation of water injection in 2D fractured media using discretefracture model, SPE Reserv. Eval. Eng. 4 (2003) 117-126.

[34] C.T. Kelley, Iterative Methods for Linear and Nonlinear Equations, SIAM, 1995.

[35] J.G. Kim, M.D. Deo, Finite element, discrete-fracture model for multiphase flow in porous media, AIChE J. 46 (2000) 1120-1130.

[36] K.A. Lie, An Introduction to Reservoir Simulation Using MATLAB: User guide for the Matlab Reservoir Simulation Toolbox (MRST), SINTEF ICT, Norway, 2014.

[37] V. Martin, J. Jaffré, J.E. Roberts, Modeling fractures and barriers as interfaces for flow in porous media, SIAM J. Sci. Comput. 26 (2005) 1667-1691

[38] S. Mishra, J. Jaffré, On the upstream mobility scheme for two-phase flow in porous media, Computat. Geosci. 14 (2010) 105-124.

[39] J. Mondteagudu, A. Firoozabadi, Control-volume model for simulation of water injection in fractured media: incorporating matrix heterogeneity and reservoir wettability effects, SPE J. 12 (2007) 355-366.

[40] F. Morales, R. Showalter, The narrow fracture approximation by channeled flow, J. Math. Anal. Appl. 365 (2010) 320-331.

[41] J.C. Nédélec, Mixed finite elements in $\mathbb{R}^{3}$, Numer. Math. 35 (1980) 315-341.

[42] Pop Iuliu Sorin, Bogers Jeroen, Kumar Kundan, Analysis and Upscaling of a Reactive Transport Model in Fractured Porous Media with Nonlinear Transmission Condition, Vietnam Journal of Mathematics (2016) 1-26. doi:10.1007/s10013-016-0198-7.

[43] V. Reichenberger, H. Jakobs, P. Bastian, R. Helmig, A mixed-dimensional finite volume method for two-phase flow in fractured porous media, Adv. Water Resour. 29 (2006) 1020-1036.

[44] J.E. Roberts, J.M. Thomas, Mixed and hybrid methods, in: P. Ciarlet, J.L. Lions (Eds.), Handbook of Numerical Analysis, volume 2, Elsevier, 1991, pp. 523-639.

[45] X. Tunc, I. Faille, T. Gallouët, M.C. Cacas, P. Havé, A model for conductive faults with non-matching grids, Computat. Geosci. 16 (2012) 277-296.

[46] C.J. Van Duijn, J. Molenaar, M.J. De Neef, The effect of capillary forces on immiscible two-phase flow in heterogeneous porous media, Transport in Porous Media 21 (1995) 71-93. 
[47] M. Vohralík, B.I. Wohlmuth, Mixed finite element methods: implementation with one unknown per element, local flux expressions, positivity, polygonal meshes, and relations to other methods, Math. Mod. Meth. Appl. Sci. 23 (2013) 803-838.

[48] A. Younès, P. Ackerer, G. Chavent, From mixed finite elements to finite volumes for elliptic PDEs in two and three dimensions, Int. J. Numer. Meth. Eng. 59 (2004) 365-388. 